\title{
Pharmacological induction of hypoxia-inducible transcription factor ARNT attenuates chronic kidney failure
}

\author{
Björn Tampe, ${ }^{1}$ Désirée Tampe, ${ }^{1}$ Gunsmaa Nyamsuren, ${ }^{1}$ Friederike Klöpper, ${ }^{1}$ Gregor Rapp, ${ }^{1}$ Anne Kauffels, ${ }^{2}$ Thomas Lorf, ${ }^{2}$ \\ Elisabeth M. Zeisberg, ${ }^{3,4}$ Gerhard A. Müller, ${ }^{1}$ Raghu Kalluri, ${ }^{5}$ Samy Hakroush, ${ }^{6}$ and Michael Zeisberg ${ }^{1,4}$ \\ ${ }^{1}$ Department of Nephrology and Rheumatology, ${ }^{2}$ Department of General, Visceral, and Pediatric Surgery, and ${ }^{3}$ Department of Cardiology and Pneumology, University Medical Center Cöttingen, \\ Georg August University, Göttingen, Germany. ${ }^{4}$ German Center for Cardiovascular Research (DZHK), Cöttingen, Germany. ${ }^{5}$ Department of Cancer Biology and the Metastasis Research Center,

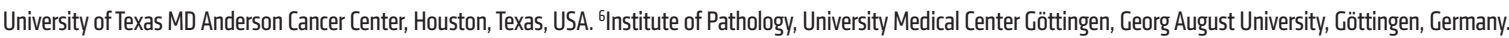

\begin{abstract}
Progression of chronic kidney disease associated with progressive fibrosis and impaired tubular epithelial regeneration is still an unmet biomedical challenge because, once chronic lesions have manifested, no effective therapies are available as of yet for clinical use. Prompted by various studies across multiple organs demonstrating that preconditioning regimens to induce endogenous regenerative mechanisms protect various organs from later incurring acute injuries, we here aimed to gain insights into the molecular mechanisms underlying successful protection and to explore whether such pathways could be utilized to inhibit progression of chronic organ injury. We identified a protective mechanism controlled by the transcription factor ARNT that effectively inhibits progression of chronic kidney injury by transcriptional induction of ALK3, the principal mediator of antifibrotic and proregenerative bone morphogenetic protein-signaling (BMP-signaling) responses. We further report that ARNT expression itself is controlled by the FKBP12/YY1 transcriptional repressor complex and that disruption of such FKBP12/YY1 complexes by picomolar FK506 at subimmunosuppressive doses increases ARNT expression, subsequently leading to homodimeric ARNT-induced ALK3 transcription. Direct targeting of FKBP12/YY1 with in vivo morpholino approaches or small molecule inhibitors, including GPI-1046, was equally effective for inducing ARNT expression, with subsequent activation of ALK3-dependent canonical BMP-signaling responses and attenuated chronic organ failure in models of chronic kidney disease, and also cardiac and liver injuries. In summary, we report an organ-protective mechanism that can be pharmacologically modulated by immunophilin ligands FK506 and CPI-1046 or therapeutically targeted by in vivo morpholino approaches.
\end{abstract}

\section{Introduction}

Progression of chronic kidney disease (CKD) toward end-stage renal disease (ESRD) is an unmet biomedical challenge, just like chronic progressive failure of any other parenchymal organ, because effective therapies to target progressive loss of functional parenchymal cells and organ fibrosis are not yet available. While efforts to implement interventional therapeutic regimens failed to treat already established chronic kidney lesions, it has been known for decades that parenchymal organs, including the kidney, can be preconditioned to resist later ensuing tissue injuries, preventing progressive loss of functional epithelium and kidney fibrosis (1-3). Based on the organ-spanning effectiveness of preconditioning regimens, we hypothesized that the underlying mechanisms could be utilized to aid the kidney in realizing its endogenous regenerative capacity even after manifestation of chronic kidney lesions.

Authorship note: BT and DT contributed equally to this work. Conflict of interest: The authors have declared that no conflict of interest exists. Submitted: July 18, 2016; Accepted: April 12, 2018.

Reference information: / Clin Invest. 2018;128(7):3053-3070.

https://doi.org/10.1172/JCl89632
Among different preconditioning approaches, several independent studies highlighted efficacy of FK506 (synonym, tacrolimus or fujimycin) administration to protect against acute experimental injuries (4) involving various parenchymal organs, including kidney (5), heart (6), liver (7), lung (8), brain (9), spinal cord (10), skin (11), and intestine (12). FK506 is a macrolide calcineurin inhibitor (CNI) that elicits immunosuppression by forming a complex with distinct FK506-binding proteins (FKBPs) to attenuate transcription of proinflammatory cytokines dependent on nuclear factor of activated T cells (NFAT) and NF-кB (13). FK506 is commonly used to lower risk of rejection after allogeneic organ transplantation, although its clinical utility is limited by its nephrotoxicity (14). While possible renoprotection by FK506 appears counterintuitive in light of its clinically established nephrotoxicity, effective organ protection was demonstrated at doses far below concentrations required for calcineurin-dependent immunosuppression (picomolar versus nanomolar range) (5-9), suggesting involvement of calcineurin-independent and yet-unknown mechanisms. Here, we aim to elucidate molecular mechanisms underlying such protective activity of low-dose FK506 administration and to explore whether this pathway could be utilized even to protect from chronic progressive organ failure. 
A

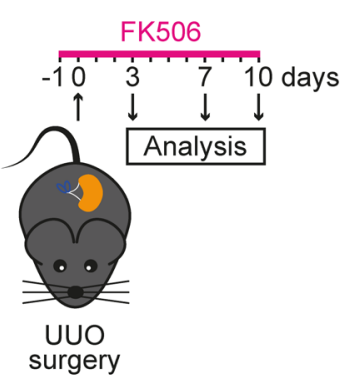

B

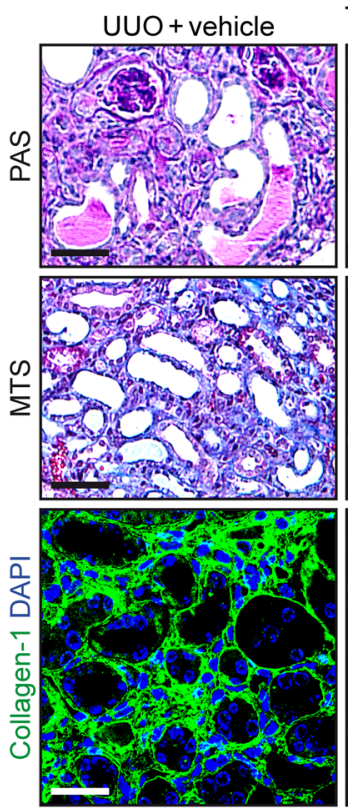

$\mathrm{UUO}+\mathrm{FK} 506(\mathrm{mg} / \mathrm{kg})$

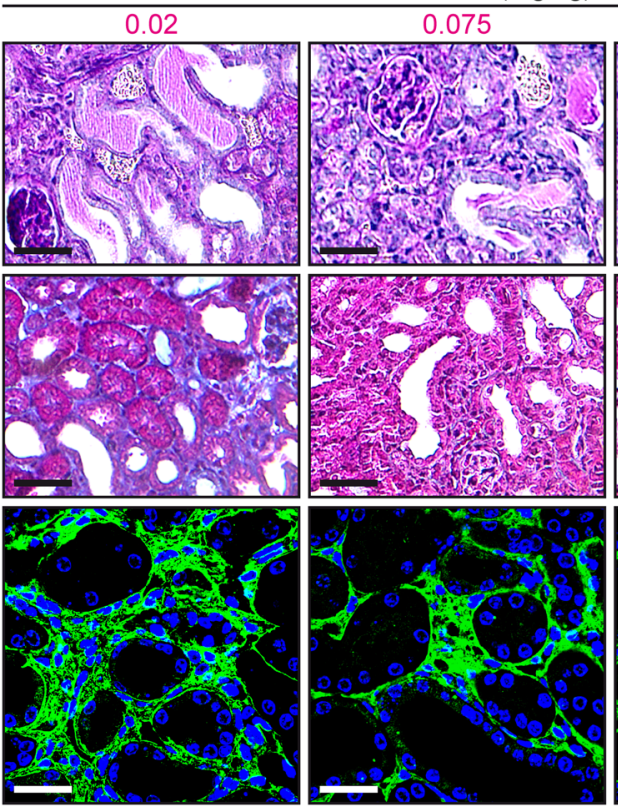

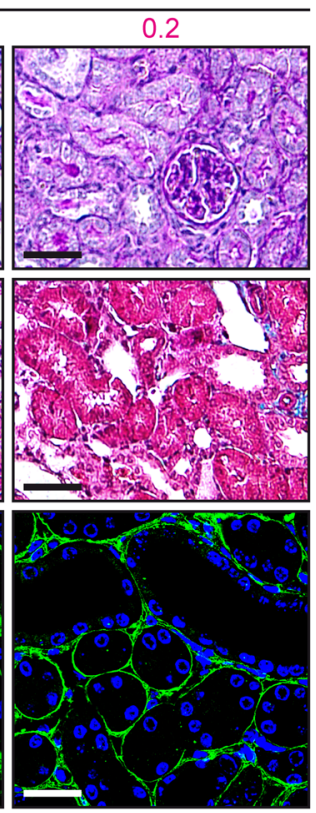

C

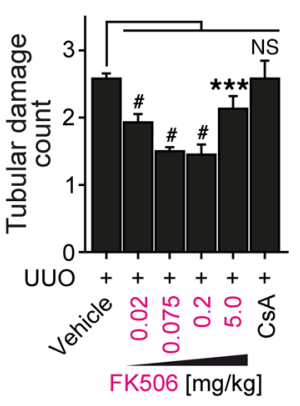

D

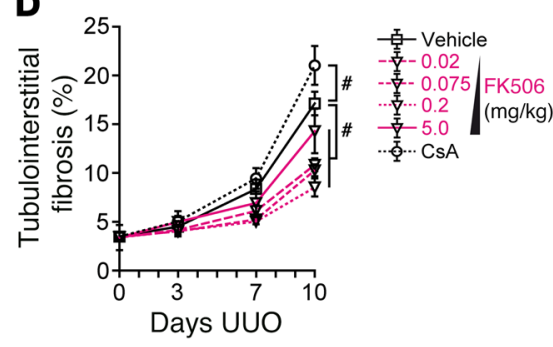

E

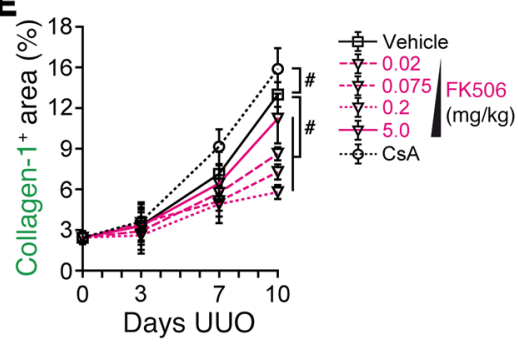

$\mathbf{F}$

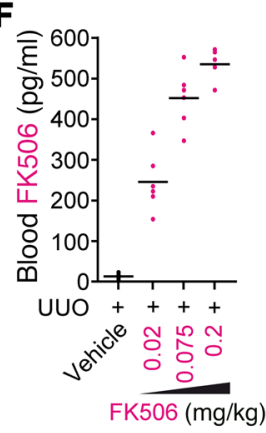

Figure 1. Low-dose FK506 protects the kidney from chronic organ injury. (A) Mice were challenged with UUO and treated with either vehicle buffer, FK506 $(0.02,0.075,0.2$, and $5.0 \mathrm{mg} / \mathrm{kg}$ orally per day, respectively), or CsA (10 mg/kg orally per day) starting 1 day before surgery. (B) The panels show representative photomicrographs of kidney sections stained with periodic acid-Schiff (PAS) and sections immunolabeled with primary antibodies against collagen-1. Scale bars: $50 \mu \mathrm{m}$ (PAS, MTS); $25 \mu \mathrm{m}$ (collagen-1). MTS, Masson's trichrome stain. (C) Tubular damage at day 10 after UUO was semiquantitatively scored using PAS-stained kidney sections. 0 , healthy; 1 , mild; 2 , moderate; 3 , severe. $n=6 /$ group. Data are presented as mean \pm SD. ${ }^{* * *} P<0.001$; $P<0.0001$, 1-way ANOVA with Bonferroni's post hoc analysis. (D) Graph summarizes average means of relative tubulointerstitial fibrosis 3, 7, and 10 days after UUO. $n=6$ /group. Data are presented as mean \pm SD. ${ }^{P} P<0.0001$, 1-way ANOVA with Bonferroni's post hoc analysis. (E) In mice receiving FK506, areas positive for collagen-1 were assessed. $n=6$ /group. Data are presented as mean \pm SD. ${ }^{\#} P<0.0001$, 1 -way ANOVA with Bonferroni's post hoc analysis. (F) FK506 concentrations were measured in whole blood samples of UUO mice receiving either vehicle buffer or FK506 $(0.02,0.075,0.2 \mathrm{mg} / \mathrm{kg}$ orally per day, respectively) using colorimetric ELISA measurements compared with standards. $n=6 /$ group. Data are presented as aligned dot plots with means.

\section{Results}

Low-dose FK506 protects the kidney from chronic organ injury dependent on enhanced ALK3 signaling. Based on previous regimens (5-9), we administered low-dose FK506 (0.02, 0.075, and 0.2 $\mathrm{mg} / \mathrm{kg}$ orally per day) to mice starting 1 day prior to challenge with unilateral ureteral obstruction (UUO) (Figure 1A). We also included standard immunosuppressive dose FK506 $(5.0 \mathrm{mg} / \mathrm{kg}$ orally per day) and cyclosporine A (CsA) (10 mg/kg orally per day), an alternative CNI that acts independently of FKBPs $(15,16)$, as controls. Histopathological analysis demonstrated that low-dose FK506 reduced both chronic tubular injury and interstitial fibrosis with an optimum dose of 0.075 and $0.2 \mathrm{mg} / \mathrm{kg}$ FK506 per day (Figure 1, B-E, and Supplemental Figure 1, A-C; supplemental material available online with this article; https://doi.org/10.1172/
JCI89632DS1). In contrast, CsA failed to attenuate tubular injury or interstitial fibrosis (Figure 1, B-E, and Supplemental Figure 1, A-C), suggesting that observed renoprotective efficacy of lowdose FK506 was mediated by mechanisms independently of calcineurin phosphatase inhibition. Administration of low-dose FK506 $(0.02,0.075$, and $0.2 \mathrm{mg} / \mathrm{kg}$ orally per day) resulted in picomolar, subimmunosuppressive FK506 blood concentrations of $245 \pm$ $73,452 \pm 71$, and $535 \pm 36 \mathrm{pg} / \mathrm{ml}$, respectively (Figure $1 \mathrm{~F}$ ). To gain insights into underlying mechanisms, we next performed gene set enrichment analysis of transcriptional expression data sets for bioactive small molecules (NCBI's Gene Expression Omnibus database [GEO] GSE5258), providing evidence that FK506 induces expression of genes involved in bone morphogenetic protein (BMP) signaling responses (Gene Ontology Consortium 


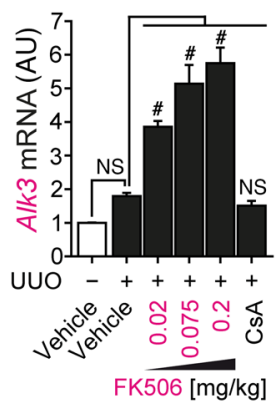

B

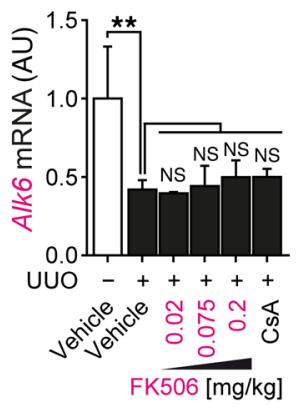

C

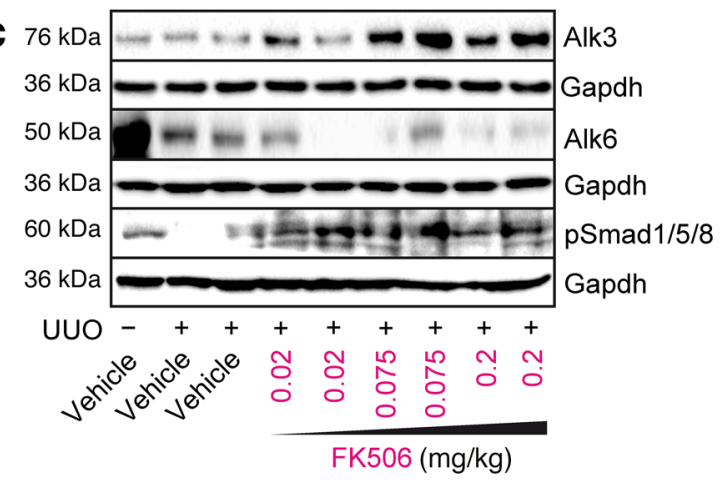

D

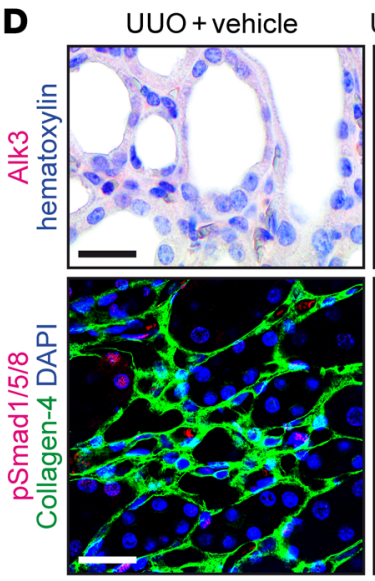

$\mathrm{UUO}+\mathrm{FK} 506(0.2 \mathrm{mg} / \mathrm{kg})$

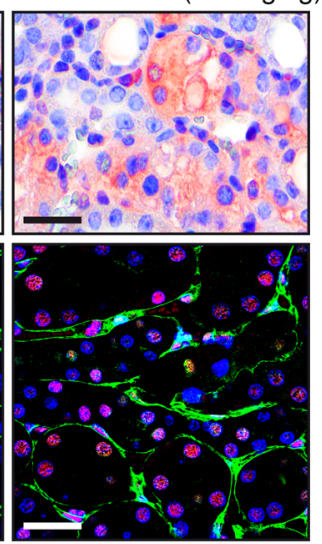

G
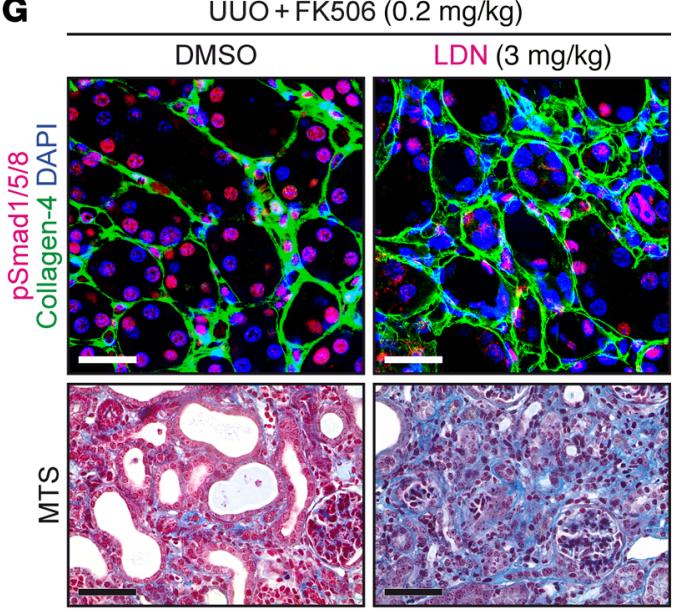

E

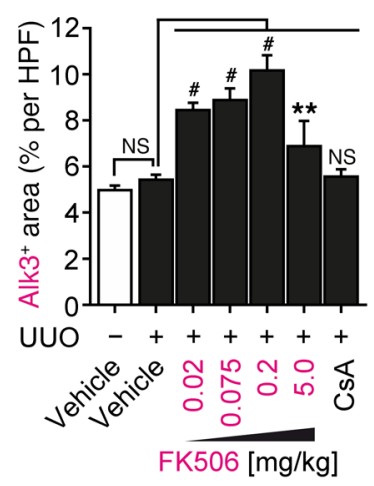

H

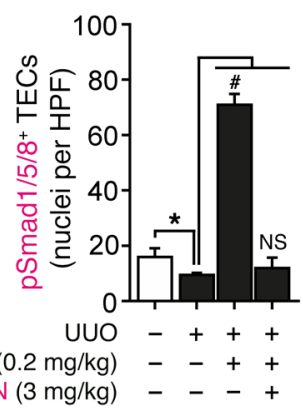

$\mathbf{F}$

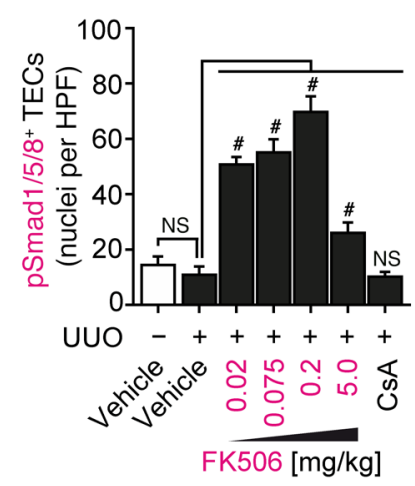

I

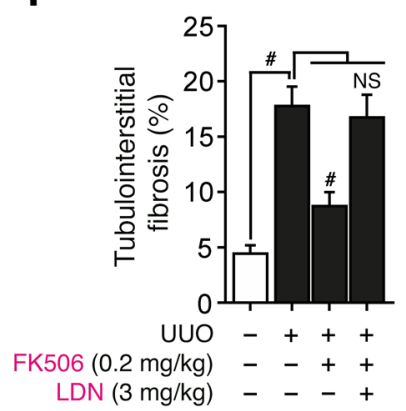

Figure 2. FK506-mediated renoprotection depends on ALK3-dependent signaling responses. (A and B) Mice were challenged with UUO and treated with either vehicle buffer or indicated concentrations of FK506 or CsA starting 1 day prior to surgery. Analysis was performed by qRT-PCR 10 days after UUO. Bar graphs reflect relative mRNA expression levels of type I BMP receptors Alk3 and Alk6. $n=3$ /group. Data are presented as mean \pm SD. ${ }^{* *} P<0.01 ;{ }^{\#} P$ $<0.0001$, 1-way ANOVA with Bonferroni's post hoc analysis. (C-F) Analysis was performed by immunoblotting of total kidney lysates and immunostaining. Type I BMP receptor Alk3 and p-Smad1/5/8 were assessed. Scale bars: $25 \mu \mathrm{m} . n=6 /$ group. Data are presented as mean \pm SD. ${ }^{* *} P<0.01 ;{ }^{*} P<0.0001$, 1 -way ANOVA with Bonferroni's post hoc analysis. See complete unedited blots in the supplemental material. (G-I) Mice were challenged with UUO and treated with either vehicle buffer or low-dose FK506 ( $0.2 \mathrm{mg} / \mathrm{kg}$ orally per day) when specifically canonical p-Smad1/5/8-dependent ALK3 signaling transduction was pharmacologically blocked with small molecule LDN ( $3 \mathrm{mg} / \mathrm{kg}$ intraperitoneally per day). Panels show representative photomicrographs of

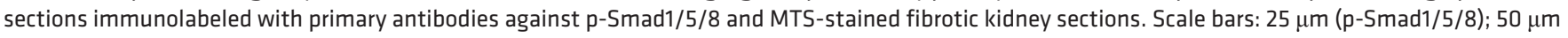
(MTS). $n=6$ /group. Data are presented as mean \pm SD. ${ }^{*} P<0.05$; ${ }^{*} P<0.0001,1$-way ANOVA with Bonferroni's post hoc analysis.

GO:0071772 and GO:0071773, Supplemental Figure 2A and Supplemental Table 1) $(17,18)$, including the prototypical type I BMP receptor ALK3 (synonym, type I BMP receptor serine/threonine kinase, BMPR1A). Because multiple independent studies dem- onstrated that administration of Alk3 ligands protected against fibrosis and loss of functional parenchymal cells in various organs including kidney (19-26), we aimed to explore a possible causal link between low-dose FK506 and Alk3-mediated renoprotection. 
Analysis of UUO-challenged murine kidneys revealed that FK506 specifically induced expression of Alk3, but not of related type I BMP receptor Alk6 (Figure 2, A-C). FK506-induced Alk3 expression was associated with nuclear phosphorylated Smad1/5/8 (p-Smad1/5/8) accumulation (correlating with protective efficacy of low-dose FK506; Figure 2, D-F, and Supplemental Figure 2B), whereas nonprotective CsA failed to induce Alk3 expression and $\mathrm{p}$-Smad1/5/8 (correlating with failure of CsA to protect injured kidneys; Figure 2, E and F, and Supplemental Figure 2B). Inhibition of canonical BMP-signaling responses by administration of dorsomorphin derivate small molecule LDN-193189 (LDN) (3 $\mathrm{mg} / \mathrm{kg}$ intraperitoneally per day) completely blocked FK506mediated renoprotection (Figure 2, G-I, and Supplemental Figure 2, C-E), confirming that FK506 elicits protection from chronic organ failure by enhanced Alk3 signaling (27).

YY1 links immunophilin FKBP12 and ALK3 transcription in response to $\mathrm{FK} 506$. We next aimed to gain insights into the molecular mechanisms underlying observed increased Alk3 transcription within tubular epithelial cells (TECs) upon low-dose FK506 administration. We exposed murine TECs to different concentrations of FK506 ranging from standard nanomolar dosages used for immunosuppression in transplant patients (2-200 nM) down to picomolar concentrations $(0.02-0.2 \mathrm{nM})$ reflecting FK506 regimens, which we had used in our murine studies. FK506 induced optimal Alk3 transcription and p-Smad1/5/8 accumulation at concentrations from $0.2 \mathrm{nM}$ to $2 \mathrm{nM}$, whereas higher concentrations had no further enhancing effects (Figure 3, A-C, and Supplemental Figure 3, A and B). Because FK506 elicits biological function by forming a complex with distinct FKBPs, we next depleted FKBP family members Fkbp12, Fkbp25, Fkbp38, and Fkbp56 in cultured TECs (Supplemental Figure 3, C-F). Whereas knockdown of Fkbp25, Fkbp38, and Fkbp56 did not affect Alk3 transcription, depletion of Fkbp12 ( $\left.F k b p 12^{K D}\right)$ induced Alk3 transcription without further addition of FK506 to culture medium (Figure 3D). Because we did not detect direct interaction of Fkbp12 and Alk3 (Figure 3E and Supplemental Figure 3G) (8) and Fkbp12 has no DNA binding properties itself, we hypothesized that Fkbp12 alters Alk3 transcription by interacting with a to-be-determined transcriptional regulator and that FK506 affects Alk3 expression via interaction with this Fkbp12/transcriptional regulator complex. We identified enrichment of genes regulated by Yin Yang 1 (YY1) in response to FK506 (GEO GSE5258, Supplemental Figure 3H and Supplemental Table 2) $(17,18,28)$, an established FKBP12-interacting protein (Supplemental Figure 3I) (29). YY1 is a GLI-Krüppel family member known to regulate transcription based on its interaction with adapter proteins, including FKBPs (30). Coimmunoprecipitation using antibodies to Yy1 (IP: Yy1) revealed direct interaction of Yy1 and Fkbp12 in cultured TECs, while such Fkbp12/Yy1 interaction was not detectable when FK506 was added to culture medium (Figure 3F and Supplemental Figure 3J). While in untreated TECs, Fkbp12 and Yy1 were equally present and detection of Fkbp12/Yy1 complexes correlated with low Alk3 transcription, depletion of Yy1 $\left(Y y 1^{K D}\right)$ was equally effective in induction of $A l k 3$ transcription and subsequent BMP-signaling activation compared with FKBP12depleted cells $\left(F k b p 12^{K D}\right.$, Figure 3, G-I, and Supplemental Figure $3 \mathrm{~K})$. In addition, supplementation of FK506 to culture medium did not additionally induce Alk3 transcription when Yy1 had been depleted (Figure 3, G-I), indicating that FK506-mediated Alk3 transcription is primarily dependent on Yy1.

To further substantiate that tubular Yy1 controls transcriptional Alk3 repression in injured kidneys, we generated mice conditionally depleted for $Y y 1$ in TECs (referred to as $\gamma G T^{\text {ret }} ; Y y 1^{f l / f l}$, Supplemental Figures 4, A and B). In mice challenged with UUO (Figure $4 \mathrm{~A})$, conditional $Y y 1$ depletion in TECs resulted in enhanced Alk3 transcription, protection from tubular injury, and renal fibrogenesis (Figure 4, B-G). When $Y y 1$ was depleted in TECs, administration of FK506 had no additive effects (Figure 4, B-G), indicating that FK506-mediated antifibrotic and proregenerative efficacy is dependent on presence and modulation of YY1 signaling in TECs.

ARNT causally links disruption of FKBP12/YY1 complexes to increased ALK3 transcription. In control experiments in which cycloheximide (CHX) (10 $\mu \mathrm{g} / \mathrm{ml})$ was added to cell-culture medium to block protein translation, FK506 failed to induce Alk3 transcription and canonical BMP-signaling responses (nuclear $\mathrm{p}$-Smad1/5/8, Figure 5, A-C). These observations suggested that de novo translation of yet-unknown mediator(s) is required to induce Alk3 transcription after release from Fkbp12/Yy1 transcriptional repressor complexes, supported by absence of putative Yy1binding motifs within the Alk3 proximal promoter region (31). By using an unbiased, array-based approach and computational prediction of putative transcriptional factor binding sites in human TECs (31), we identified 6 candidate transcriptional factors with a binding motif within the $A L K 3$ proximal promoter at least 2-fold induced upon FK506 exposure: AR, ARNT, CEBPB, CREB1, GATA3, and MAX (Figure 5, D and E). While quantitative reversetranscriptase PCR (qRT-PCR) confirmed transcriptional induction of all identified factors upon exposure to FK506 (Figure 5F), only siRNA-mediated depletion of Arnt $\left(A r n t^{K D}\right)$ prevented transcriptional Alk3 induction in response to FK506 (Figure 5G and Supplemental Figure 5, A-F). Furthermore, we observed robust Arnt induction upon depletion of either Fkbp12 (Fkbp12 $\left.{ }^{K D}\right)$ or Yy1 $\left(Y y 1^{K D}\right.$, Figure $\left.5 \mathrm{H}\right)$, suggesting Arnt as a possible link between Fkbp12/Yy1 complex disruption and enhanced Alk3 transcription. These observations were further supported by transcriptional expression data sets with robust induction of $A R N T$ and enrichment of ARNT-regulated genes induced in response to FK506 (GEO GSE5258, Supplemental Figure 3H and Supplemental Table 2) $(17,18,28)$. To further explore a causal link between Fkbp12/ Yy1 complex disruption and increased Alk3 transcription, we performed Arnt ChIP PCR using antibodies to Yy1 for immunoprecipitation (IP: Yy1). ChIP demonstrated binding of Yy1 to its putative motif within the Arnt proximal promoter in cultured TECs that had been maintained in control media without FK506 (with low Alk3 expression levels, Figure 5I). Addition of FK506 to culture media (associated with enhanced Alk3 transcription) reduced Yy1 binding to the Arnt proximal promoter (Figure 5I) associated with transcriptional Arnt induction (Figure 5J). Supplementation of culture media with FK506 did not additionally induce Arnt mRNA levels in experiments in which Arnt had been induced by Fkbp12 $\left(F k b p 12^{K O}\right)$ or Yy1 depletion (Yy $1^{K O}$, Figure 5J), supporting the concept that FK506-mediated Arnt transcription is primarily dependent on Fkbp12/Yy1.

To further substantiate the causal link between Arnt and Alk3 regulation, we next analyzed efficacy of FK506 to enhance Alk3 
A

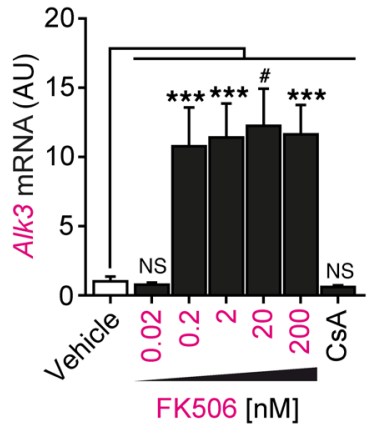

B

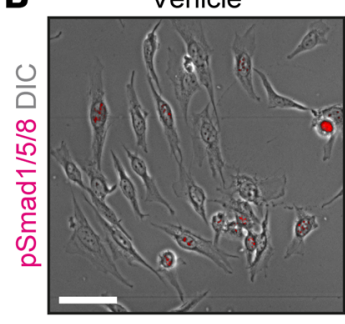

FK506 (200 pM)

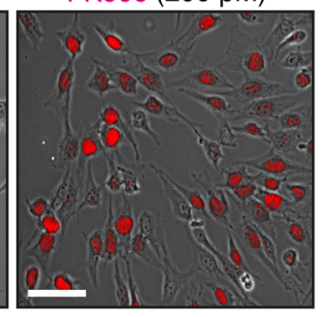

C

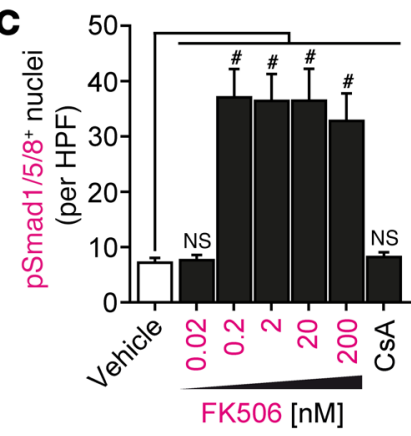

D

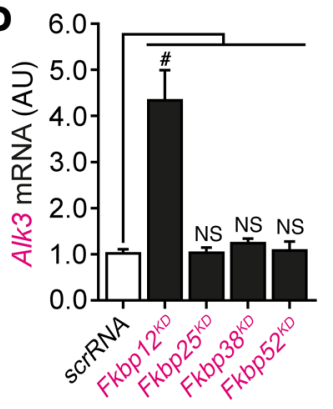

E

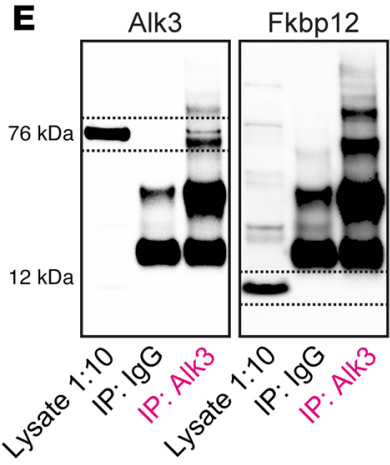

G

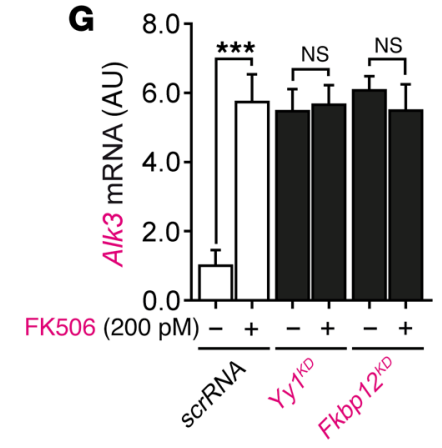

H

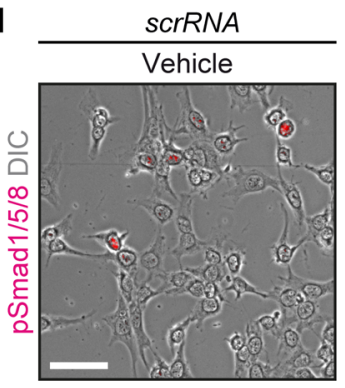

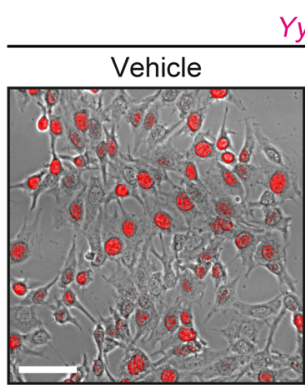

$Y y 1^{K D}$

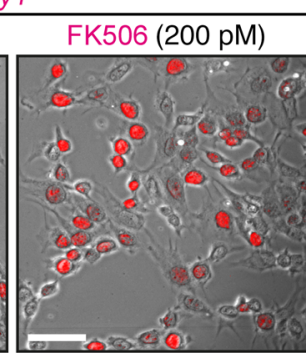

$\mathbf{F}$

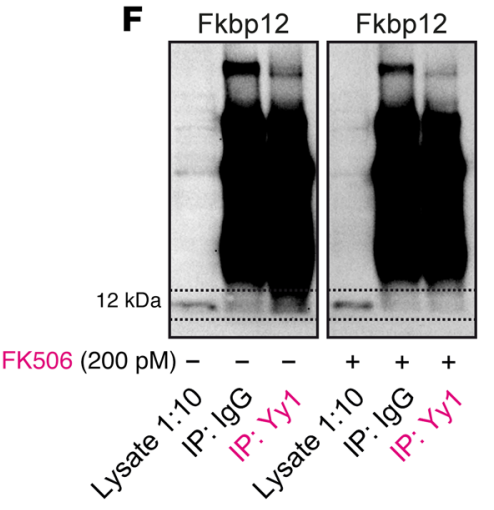

FK506 (200 pM) -

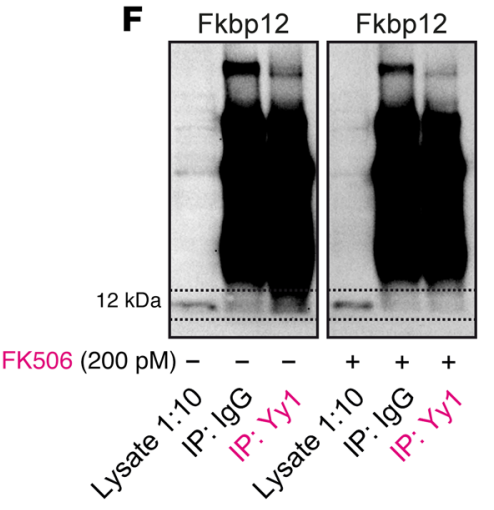

FK506 (200 pM)

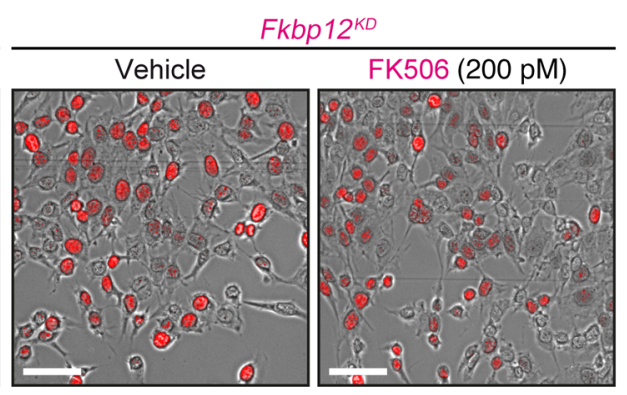

I

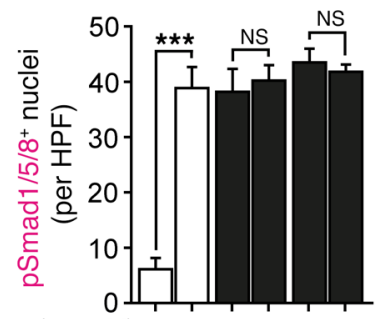

FK506 (200 pM)

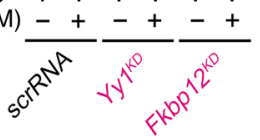

Figure 3. FK506 disrupts an FKBP12/YY1 transcriptional repressor complex. (A) TECs were exposed to vehicle, indicated concentrations of FK506 (0.02, $0.2,2,20,200 \mathrm{nM}$, respectively), or equimolar CsA (10 nM). mRNA expression levels of Alk3 were analyzed by qRT-PCR. $n=3$ independent experiments. Data are presented as mean \pm SD. ${ }^{* *} P<0.001 ;{ }^{*} P<0.0001,1$-way ANOVA with Bonferroni's post hoc analysis. (B and C) Representative photomicrographs of $\mathrm{p}-\mathrm{Smad1} / 5 / 8$ complex ( $\mathrm{p}-\mathrm{Smad1/5/8}$ ) immunostainings overlayed with differential interference contrast (DIC) are shown. Scale bars: $25 \mu \mathrm{m}$. $n=3$ independent experiments. Data are presented as mean \pm SD. ${ }^{P} P<0.0001,1$-way ANOVA with Bonferroni's post hoc analysis. (D) Alk3 mRNA expression levels in TECs were analyzed by qRT-PCR after siRNA-mediated knockdown of Fkbp12 (Fkbp12 $\left.{ }^{K D}\right)$, Fkbp25 (Fkbp25 $\left.{ }^{K D}\right)$, Fkbp38 $\left(F k b p 38^{K D}\right)$, or Fkbp56 (Fkbp56 ${ }^{K D}$. $n=$ 3 independent experiments. Data are presented as mean \pm SD. ${ }^{*} P<0.001,1$-way ANOVA with Bonferroni's post hoc analysis. (E) As analyzed by coimmunoprecipitation after Alk3 pulldown (IP: Alk3), direct interaction between Fkbp12 and Alk3 was assessed. (F) As analyzed by coimmunoprecipitation after Yy1 pulldown (IP: Yy1), direct interaction between Yy1 and Fkbp12 was assessed. See complete unedited blots in the supplemental material. (G) Alk3 mRNA expression levels were assessed by qRT-PCR after knockdown of either Yy1 ( $\left.Y y 7^{K D}\right)$ or Fkbp12 (Fkbp12 $\left.{ }^{K D}\right)$ and exposure to FK506. $n=3$ independent experiments. Data are presented as mean \pm SD. ${ }^{* *} P<0.001$, Student's $t$ test. (H and I) Representative photomicrographs of $p-S m a d 1 / 5 / 8$ immunostainings overlayed with DIC are shown. Scale bars: $25 \mu \mathrm{m} . n=3$ independent experiments. Data are presented as mean \pm SD. ${ }^{* * *} P<0.001$, Student's $t$ test. 
A

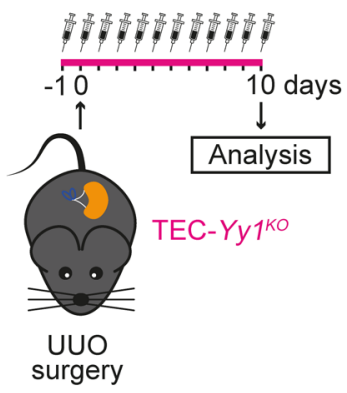

B

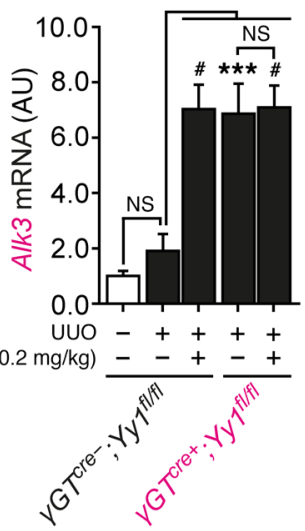

D

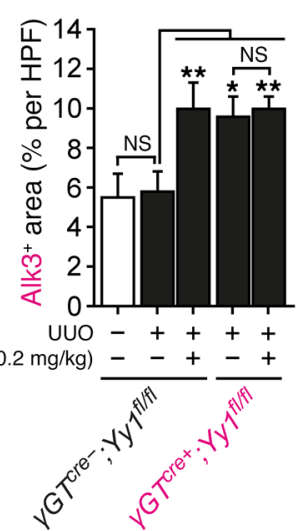

C

\section{(2)}

UUO: $\gamma G T^{\text {cre }} ; Y y y 1^{f l f f l}$
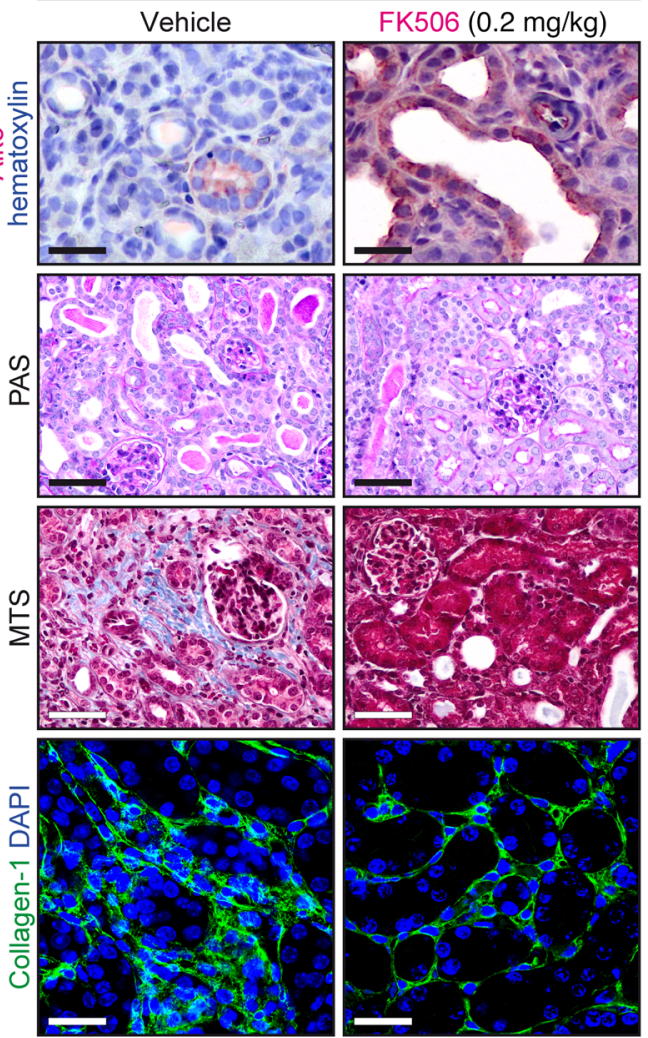

E

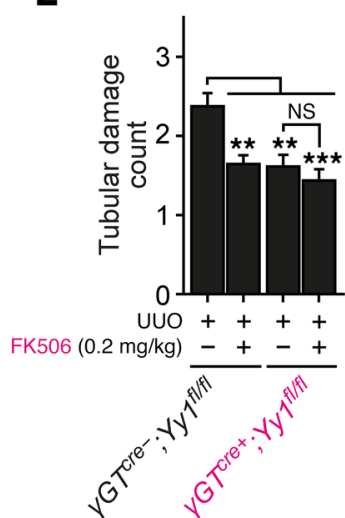

$\mathbf{F}$

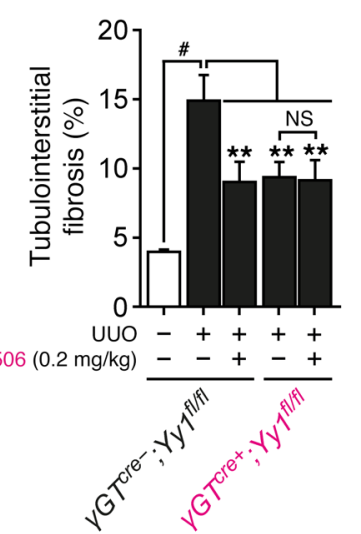

UUO: $y G^{\text {cre+;}}$ Yyy $1^{f l f l}$

Vehicle FK506 (0.2 mg/kg)
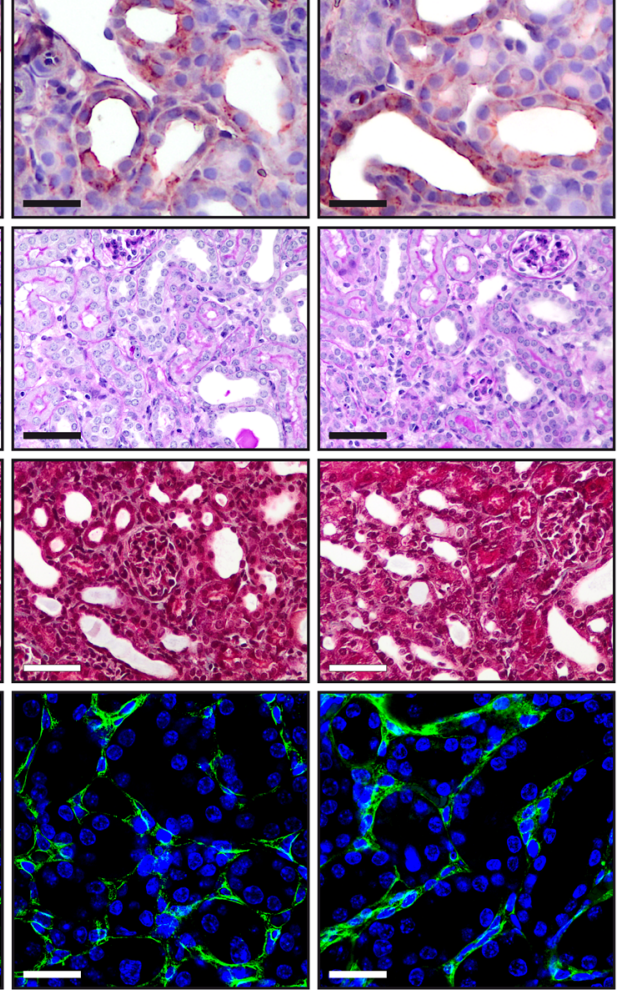

G

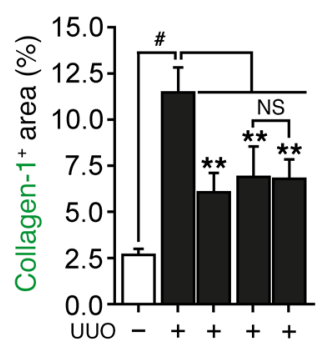

$\mathrm{FK} 506(0.2 \mathrm{mg} / \mathrm{kg})-\mathbf{+}+\mathbf{+}+$

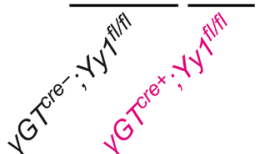

Figure 4. FK506-mediated protection is dependent on presence and modulation of YY1 signaling in TECs. (A) Mice conditionally depleted for YY1 in TECs $\left(\gamma \in T^{\text {cret }} ; Y y 7^{f / f f}\right)$ and corresponding littermate controls $\left(\gamma G T^{\text {cre- }} ; Y y 7^{f l / f f}\right)$ were challenged with UUO and treated with either vehicle buffer or FK506 (0.2 mg/kg orally per day) starting 1 day prior to surgery. (B) Alk3 mRNA expression levels were analyzed by qRT-PCR. $n=3 /$ group. Data are presented as mean \pm SD. ${ }^{* * *} P<0.001$; ${ }^{*} P<0.0001$, 1-way ANOVA with Bonferroni's post hoc analysis. (C-C) Representative photomicrographs of immunostainings for Alk3, PASstained fibrotic kidney sections, MTS, and collagen-1 in mice challenged with UUO are shown. Scale bars: $25 \mu \mathrm{m}$ (Alk3); $50 \mu \mathrm{m}$ (PAS); $50 \mu \mathrm{m}$ (MTS); $25 \mu \mathrm{m}$ (collagen-1). $n=3$ /group. Data are presented as mean $\pm \mathrm{SD}$. ${ }^{*} P<0.05$; ${ }^{* *} P<0.01 ;{ }^{* * *} P<0.001 ;{ }^{\#} P<0.0001$, 1-way ANOVA with Bonferroni's post hoc analysis.

transcription when Arnt was blocked in cultured TECs $\left(A r n t^{K D}\right)$. When Arnt induction was depleted, FK506 failed to induce Alk3 transcription (Figure 6A and Supplemental Figure 5B). In addition, transgenic Arnt overexpression $\left(A r n t^{\circ c}\right)$ alone was sufficient to specifically induce Alk3 transcription (Figure 6B and Supplemental Figure 5, G and H). Generally, ARNT (synonym, HIF1 $\beta$ ) is a member of the PAS domain family and forms heterodimeric transcription factors with other PAS family members (HIF1 $\alpha$ in hypoxia responses and the dioxin receptor AHR in xenobiotic sig- naling) or homodimers to elicit transcriptional activation (by binding to the E-box core sequence CACGTG) (32). FK506 exposure did not markedly induce pathways involved in hypoxic signaling or drug metabolism (including xenobiotic signaling; Figure 6, C and D), and coimmunoprecipitation using antibodies to Arnt (IP: Arnt) did not provide evidence for Arnt/Hifl $\alpha$ or Arnt/Ahr interaction in cultured TECs in response to FK506 (Figure 6, E-G). These observations were further confirmed by preserved effectiveness of FK506 or transgenic Arnt overexpression to induce Alk3 tran- 
A

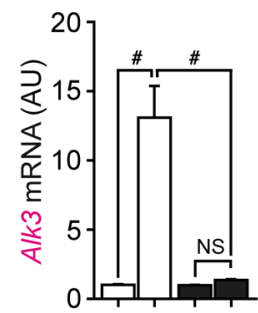

FK506 (200 pM) - + - +

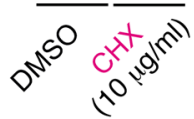

B

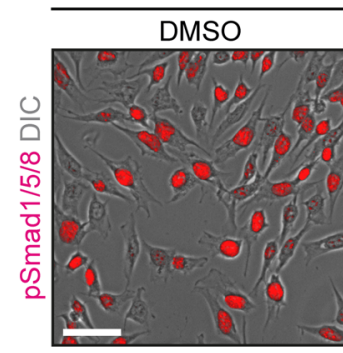

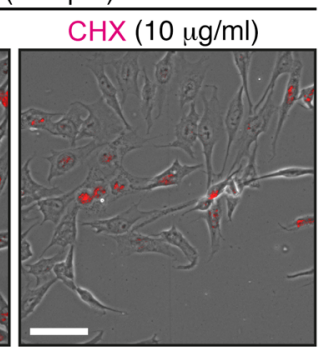

C

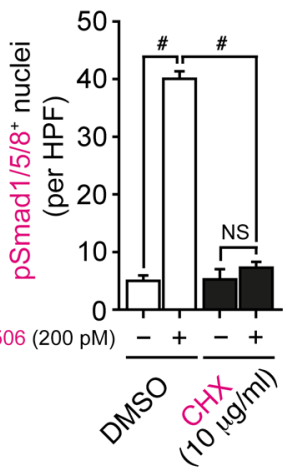

E

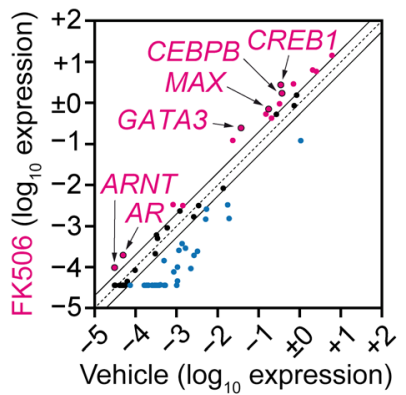

H

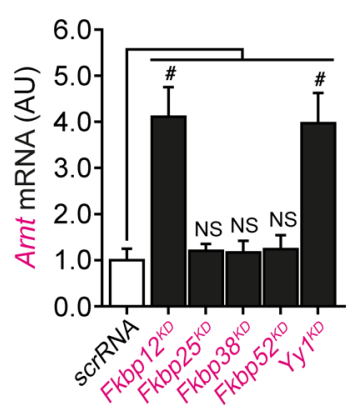

$\mathbf{F}$

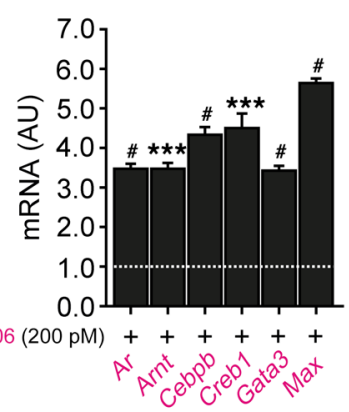

I

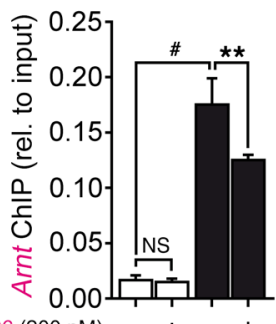

FK506 (200 pM)

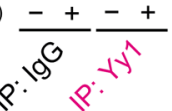

G

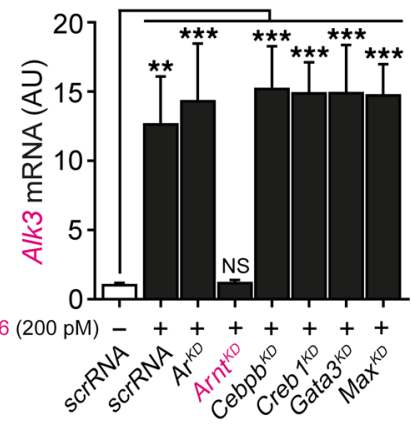

J

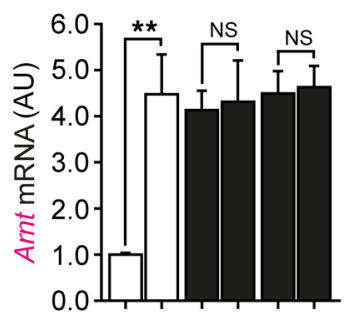

FK506 (200

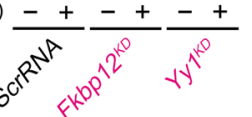

D
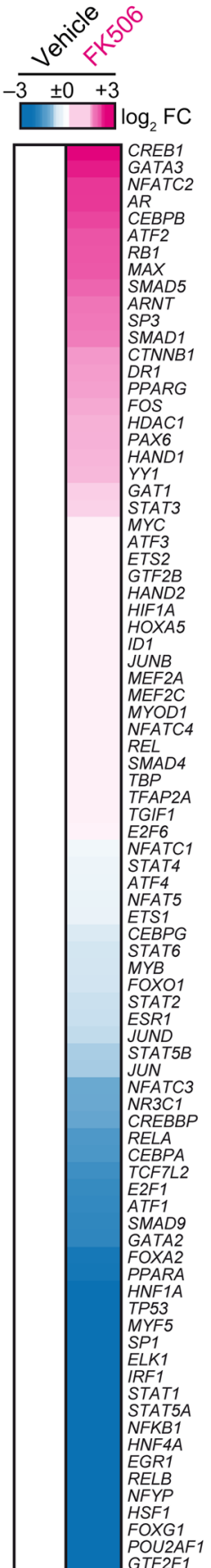

Figure 5. ARNT causally links disruption of FKBP12/YY1 complexes to enhanced ALK3 transcription. (A) TECs were exposed to CHX 1 hour prior to FK506 incubation. Alk3 mRNA expression was assessed by qRT-PCR. $n=3$ independent experiments. Data are presented as mean \pm SD. ${ }^{2} P<0.001,1$-way ANOVA with Bonferroni's post hoc analysis. (B and C) Immunostaining of p-Smad1/5/8 overlayed with DIC. Scale bars: $25 \mu \mathrm{m}$. $n=3$ independent experiments. Data are presented as mean \pm SD. ${ }^{P}<<0.0001$, 1-way ANOVA with Bonferroni's post hoc analysis. (D and E) FK506-mediated transcriptional network alterations; $\log _{2}$ fold changes $\left(\log _{2}\right.$ FC) are shown by heatmap. (F) Validation by qRT-PCR upon FK506 exposure. $n=3$ independent experiments. Data are presented as mean $\pm \mathrm{SD}$. ${ }^{* * *} P<0.001 ;{ }^{*} P<0.0001$, Student's $t$ test in comparison with DMSO-treated control cells. (C) Alk3 mRNA levels were analyzed by qRT-PCR. $n=3$ independent experiments. Data are presented as mean \pm SD. ${ }^{* *} P<0.01$; ${ }^{* *} P<0.001$, 1-way ANOVA with Bonferroni's post hoc analysis. (H) Arnt mRNA expression levels, as analyzed by qRT-PCR, are shown. $n=3$ independent experiments. Data are presented as mean \pm SD. ${ }^{\#} P<0.0001$, 1-way ANOVA with Bonferroni's post hoc analysis. (I) Binding of Yy1 to the Arnt proximal promoter was analyzed by ChIP after Yy1 pulldown (IP: Yy1). $n=3$ technical replicates. Data are presented as mean $\pm \mathrm{SD}$. ${ }^{* *} P<0.01$; ${ }^{*} P<0.0001,1$-way ANOVA with Bonferroni's post hoc analysis. (J) Arnt mRNA expression levels, as assessed by qRT-PCR, are shown. $n=3$ independent experiments. Data are presented as mean \pm SD. ${ }^{* *} P<0.01$, Student's $t$ test. 
A
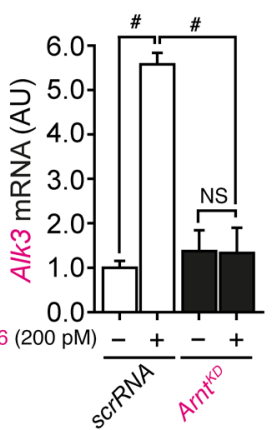

$\mathbf{E}$

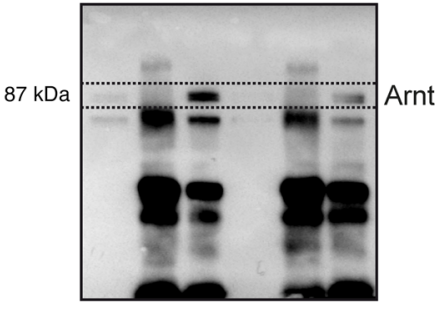

FK506 (200 pM) + + + - - -

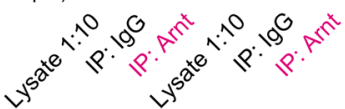

H

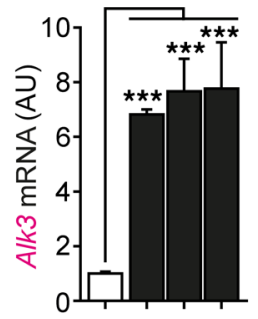

FK506 (200 pM) - + + +

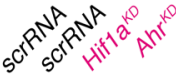

FK506 (200 pM)

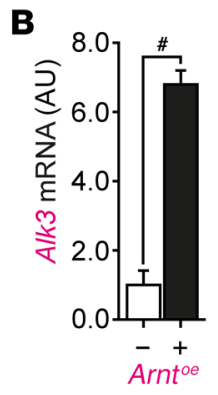

C

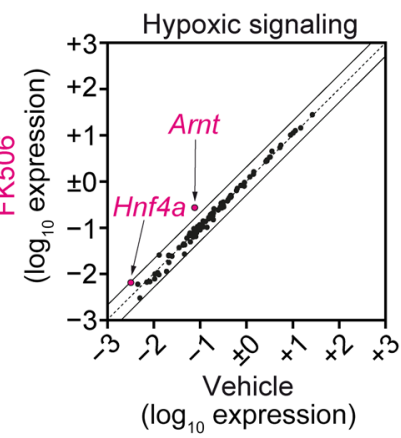

$\mathbf{F}$

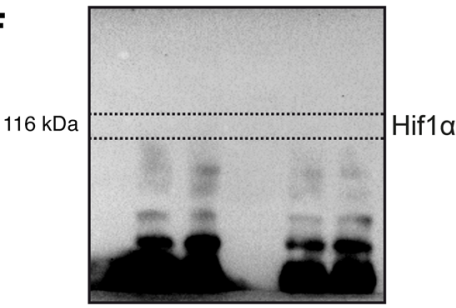

FK506 (200 pM) + + + - - -
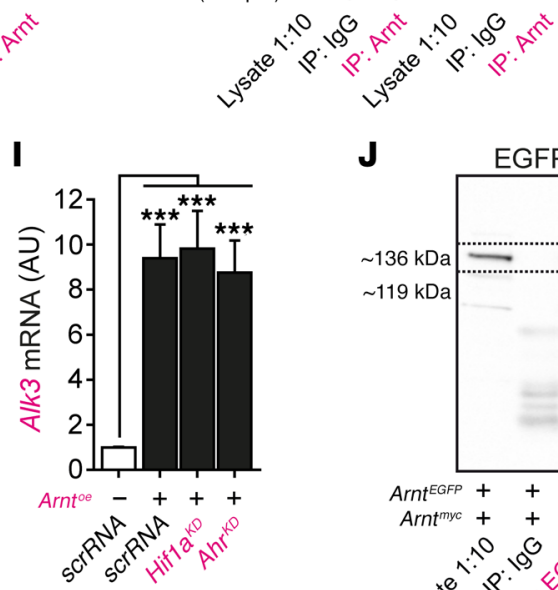

$\mathbf{L}$

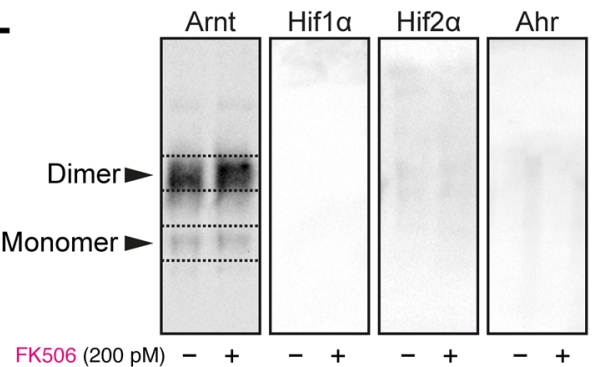

M

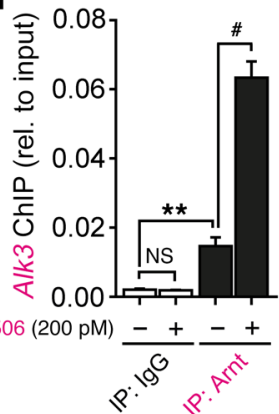

J

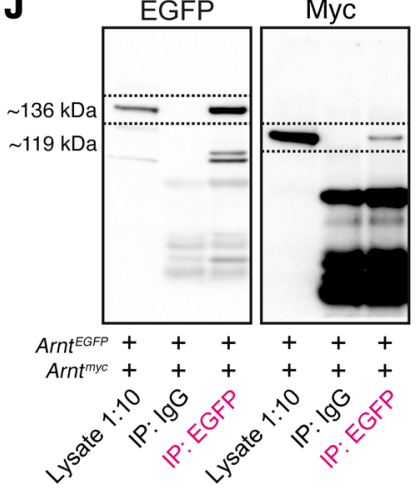

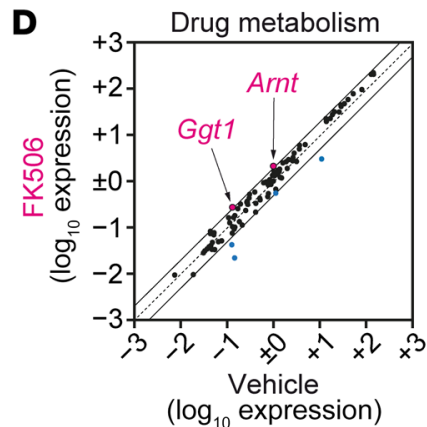

G

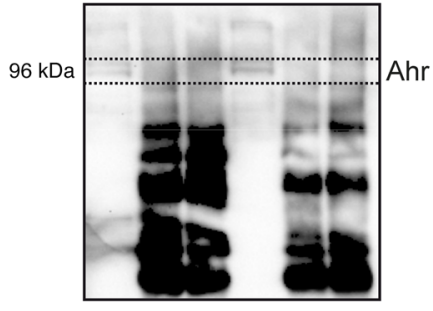

FK506 (200 pM) - - - + + +
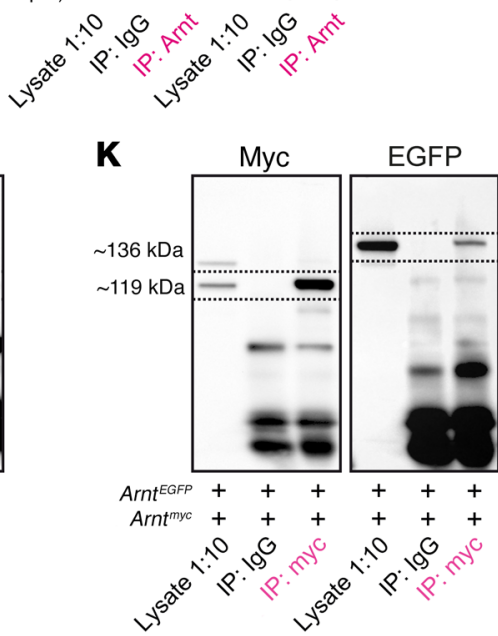

$\mathbf{N}$

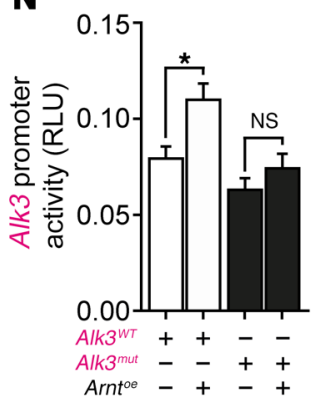

Figure 6. ARNT targets a palindromic E-box motif specific for ARNT homodimers required for ALK3 transcription. (A) Alk3 mRNA was assessed by qRT-PCR after depletion of $\operatorname{Arnt}\left(\operatorname{Arnt}{ }^{K D}\right) . n=3$ independent experiments. Data are presented as mean $\pm S D$. ${ }^{\#} P<0.0001$, 1-way ANOVA with Bonferroni's post hoc analysis. (B) Alk3 mRNA expression levels after Arnt overexpression (Arnt ${ }^{\circ e}$ ) are shown. $n=3$ independent experiments. Data are presented as mean \pm SD. ${ }^{\#} P<0.0001$, Student's $t$ test. (C and $\left.\mathbf{D}\right)$ Impact of FK506 on hypoxic signaling and drug metabolism. (E-C) Analysis was performed by coimmunoprecipitation using antibodies against Arnt (IP: Arnt), Arnt/Hif1 $\alpha$, and Arnt/Ahr. (H and I) Efficacy of FK506 or Arnt overexpression in inducing Alk3 mRNA expression levels in cultured TECs depleted for HIF1 $\alpha\left(H i f 1 a^{K D}\right)$ or AHR $\left(A h r^{K D}\right) . n=3$ independent experiments. Data are presented as mean \pm SD. ${ }^{* * *} P<0.001,1$-way ANOVA with Bonferroni's post hoc analysis. ( and $\mathbf{K}$ ) Analysis by coimmunoprecipitation. Homodimer formation was assessed in cultured TECs after EGFP-tagged (Arnt ${ }^{E C F P}$ )

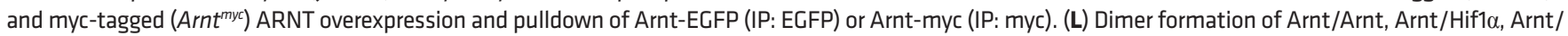
Hif2 $\alpha$ and Arnt/Ahr was assessed by native gel electrophoresis. See complete unedited blots in the supplemental material. (M) Binding of Arnt to the Alk3 proximal promoter was analyzed by ChIP and subsequent target PCR after Arnt pulldown (IP: Arnt). $n=3$ technical replicates. Data are presented as mean \pm SD. ${ }^{* *} P<0.01 ;{ }^{*} P<0.0001$, 1-way ANOVA with Bonferroni's post hoc analysis. (N) Analysis was performed by reporter assays. Alk3 proximal promoter activity was assessed in the presence (Alk3 ${ }^{W T}$ ) or absence (Alk $3^{\text {mut }}$, CACGTC to TATATA) of the palindromic E-box motif. $n=5$ independent experiments. Data are presented as mean $\pm S D .{ }^{*} P<0.05$, Student's $t$ test. 
scription when either Hifla $\left(H i f 1 a^{K D}\right)$ or Ahr $\left(A h r^{K D}\right)$ was depleted (Figure 6, H and I, and Supplemental Figure 5, I-L). To elucidate capacity of Arnt homodimer formation in cultured TECs, we next generated EGFP-tagged (Arnt $\left.t^{E G F P}\right)$ and myc-tagged $\left(A r n t^{m y c}\right)$ Arnt overexpression plasmids and confirmed formation of Arnt homodimers by coimmunoprecipitation with Arnt ${ }^{\text {myc }}$ after pulldown of Arnt ${ }^{\mathrm{EGFP}}$ (IP: EGFP) and vice versa (IP: myc; Figure 6, J and $\mathrm{K}$ ). Analysis of endogenous Arnt by native gel electrophoresis revealed the presence of Arnt dimers within TECs cultivated under standard conditions, and further enhancement resulted upon FK506 exposure (Figure 6L). We did not detect Hif1 $\alpha$, Hif $2 \alpha$, or Ahr as constituents of Arnt dimers, further suggesting that transcriptional responses to FK506 in TECs are mediated by homodimerized Arnt (Figure 6L) (32). Alk3 ChIP-PCR revealed increased Arnt binding to its respective homodimer motif upon exposure to FK506 (associated with enhanced Arnt homodimers and enhanced Alk3 transcription; Figure 6M) $(33,34)$. Transcriptional Alk3 induction was further confirmed by reporter assays, as Arnt overexpression effectively induced Alk3 proximal promoter activity (Alk3 ${ }^{W T}$, Figure $\left.6 \mathrm{~N}\right)$. In contrast, Arnt failed to induce Alk3 transcription when the Arnt homodimer target sequence was disrupted (CACGTG to TATATA, Alk3 ${ }^{m u t}$, Figure $6 \mathrm{~N}$ ).

These findings in cultured TECs were further confirmed by increased Arnt levels in $\gamma G T^{\text {rer }} ; Y y 1^{f / f l}$ conditional KO mice and in mice treated with low-dose FK506 (Figure 7, A-E, correlating with enhanced Alk3 transcription and renoprotection). Furthermore, analysis of total kidney lysates by immunoprecipitation, native gel analysis, and subsequent immunoblotting revealed enhanced formation of Arnt dimers without interaction with Hif1 $\alpha$, Hif $2 \alpha$, or Ahr (Figure $7 \mathrm{~F}$ ), confirming a critical role of Arnt homodimerization.

Selective modulation of FKBP12/YY1 signaling effectively modulates protective ARNT within chronically injured kidneys. To elucidate therapeutic implication of our findings, we next selectively modulated constituents of the identified Fkbp12/Yy1/Arnt signaling axis in mice challenged with UUO by administration of in vivo morpholinos (VMO) targeting translational start sites (Supplemental Table 3-5) (35). As compared with control-VMO targeting a human $\beta$-globin intron mutation (36), administration of VMO targeting Fkbp12 (Fkbp12-VMO), Yy1 (Yy1-VMO), or Arnt (Arnt$\mathrm{VMO}$ ) effectively reduced intrarenal protein levels of their respective transcripts (Figure 8, A and B, and Supplemental Figure 6, A-F). Administration of Fkbp12-VMO, Yy1-VMO, or FK506 equally induced intrarenal Arnt, associated with attenuated tubular injury and tubulointerstitial fibrosis (Figure 8, A-D, and Supplemental Figure 6, F-H). Administration of FK506 had no additive protective effects in cohorts of mice that had also received Fkbp12-VMO or Yy1-VMO (Figure 8, A-D, and Supplemental Figure 6, F-H). In addition, administration of Fkbp12-VMO or Yy1-VMO did not further enhance the effect of FK506 on renoprotection (Figure 8, A-D, and Supplemental Figure 6, F-H), mirroring the effect of genetic Yy1 depletion that we had observed in $\gamma G T^{\text {ret }} ; Y y 1^{f l / f l}$ conditional KO mice. In contrast, FK506 failed to protect kidneys from tubular injury and progressive fibrotic disease when intrarenal Arnt induction was depleted (Figure 8, A-D, and Supplemental Figure 6, F-H).

Selective targeting of FKBP12 by nonimmunosuppressive FKBP12 inhibitor GPI-1046 effectively modulates FKBP12/YY1/ARNT signaling and protects from chronic renal failure. Because our data sug- gested that picomolar FK506 elicited renoprotective properties independently of calcineurin inhibition, we next explored efficacy of a specific small molecule Fkbp12 inhibitor, 3-(3-pyridyl)1-propyl-(2S)-1-(3,3-dimethyl-1,2-dioxopentyl)-2-pyrrolidinedine carboxylate (GPI-1046), an FK506 derivate without immunosuppressive properties (37), to modulate intrarenal Fkbp12/Yy1/ Arnt/Alk3 signaling and to preempt chronic renal injury. Exposure of cultured TECs to GPI-1046 $(10 \mu \mathrm{M})$ was equally effective in enhancing Arnt and Alk3 transcription (Figure 9, A and B). Based on our previous regimen, we next administered GPI-1046 (10 mg/kg subcutaneously per day) 1 day prior to UUO challenge (Figure 9C) (37). Nonimmunosuppressive GPI-1046 effectively induced intrarenal Arnt and Alk3 transcription and BMP-signaling responses and protected from tubular injury and renal fibrogenesis (Figure 9, D-K), further supporting the idea that immunophilin Fkbp12 is specifically involved in modulating intrarenal Arnt/Alk3 signaling and protection from injury independently of calcineurin modulation or immunosuppressive properties. This was further confirmed by uniform immune cell infiltration in cohorts of mice administered either picomolar FK506 or nonimmunosuppressive GPI-1046 not affecting intrarenal NFAT signaling (Supplemental Figure 7, A-C). To determine whether administration of FK506 or GPI-1046 was also effective in attenuating CKD progression when administration is initiated after manifestation of kidney injury, we next administered FK506 or GPI-1046 3 days after challenging with UUO $(0.2 \mathrm{mg} / \mathrm{kg}$ or $30 \mathrm{mg} / \mathrm{kg}$ orally per day, respectively, Figure 10A). Both FK506 and small molecule Fkbp12 inhibitor GPI-1046 were equally effective in inducing intrarenal Arnt and Alk3 transcription, associated with attenuated tubular injury and renal fibrogenesis (Figure 10, B-H).

Selective targeting of FKBP12 protects from chronic injury in the heart and liver. Effectiveness of FK506 had been documented in various parenchymal organs (4-12), in line with transcriptional Arnt induction present in the kidney (confirming previous results) and also in the heart, brain, spinal cord, skin, liver, lung, and intestine in response to FK506 or GPI-1046 (0.2 mg/ $\mathrm{kg}$ or $30 \mathrm{mg} / \mathrm{kg}$ orally per day, respectively; Figure 11, A and B). Based on robust Arnt induction observed in the heart and liver (Supplemental Figure 8, A and B), we next analyzed the presence of an Fkbp12/Yy1/Arnt/Alk3 signaling axis in rodent models of cardiac fibrosis after continuous minipump delivery of angiotensin II (AT II) and carbon tetrachloride-induced $\left(\mathrm{CCl}_{4}\right.$-induced) liver failure $(23,38)$. Fkbp12 and Yy1 were detectable in chronically injured hearts and livers (Supplemental Figure 9, $\mathrm{A}-\mathrm{F}$ ), suggesting that repressive Fkbp12/Yy1 complexes were equally present. To elucidate whether the identified Fkbp12/Yy1/Arnt/ Alk3 signaling axis could be equally targeted to protect from chronic heart failure and fibrosis, we next administered GPI-1046 (10 mg/ $\mathrm{kg}$ subcutaneously per day) 1 day prior to administration of AT II (Figure 11C). GPI-1046 was equally effective in induction of Alk3dependent canonical BMP-signaling responses and protection from fibrogenesis in the heart (Figure 11, D-K, and Supplemental Figure $9, G^{-} J$ ). These results were also confirmed in mice challenged with $\mathrm{CCl}_{4}$ (Figure 12A). GPI-1046 equally induced Alk3-dependent signaling responses and protected from chronic liver failure (Figure 12, B-H, and Supplemental Figure 9, K and L).

An FKBP12/YY1/ARNT/ALK3 signaling axis translates to humans. With regard to translational implications of our find- 
A

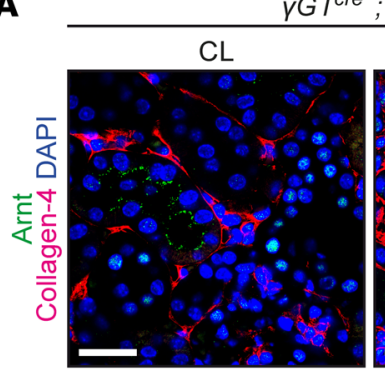

$\gamma G T^{\text {ore- }} ; Y y 1^{f / / f l}$

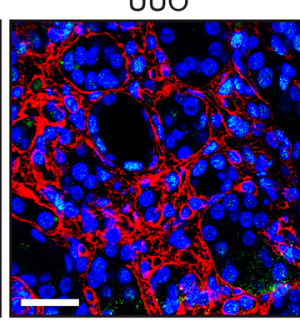

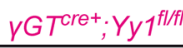

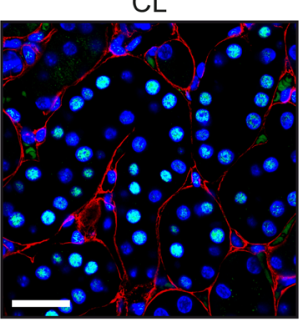

UUO

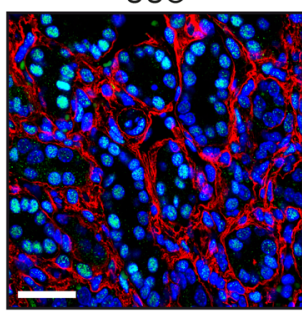

B

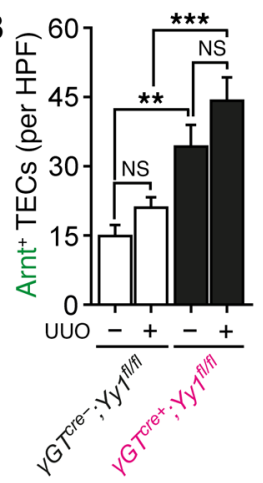

UUO + FK506 (mg/kg)
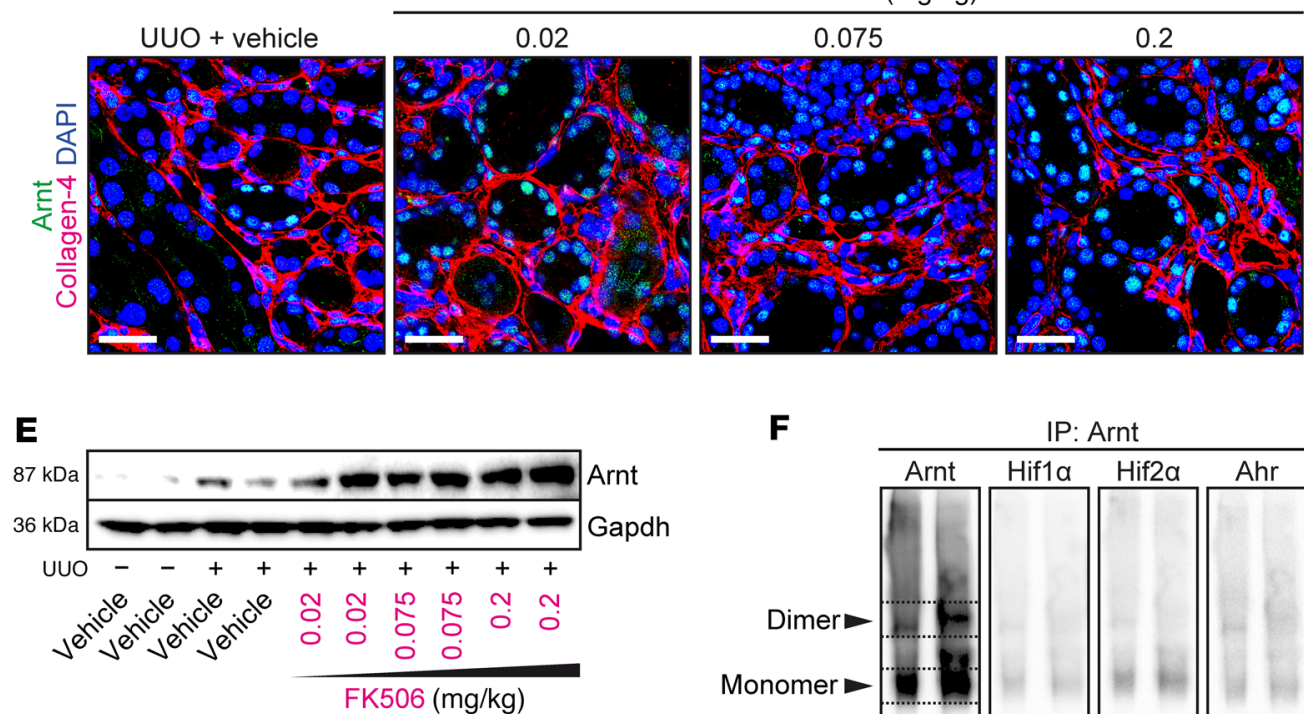

D

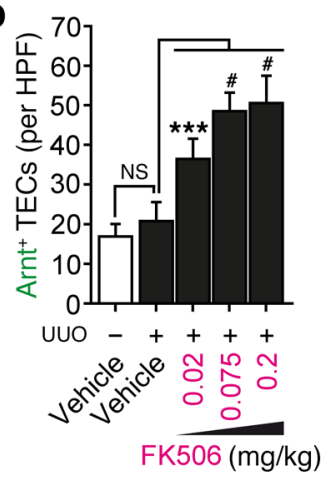

$\mathbf{F}$

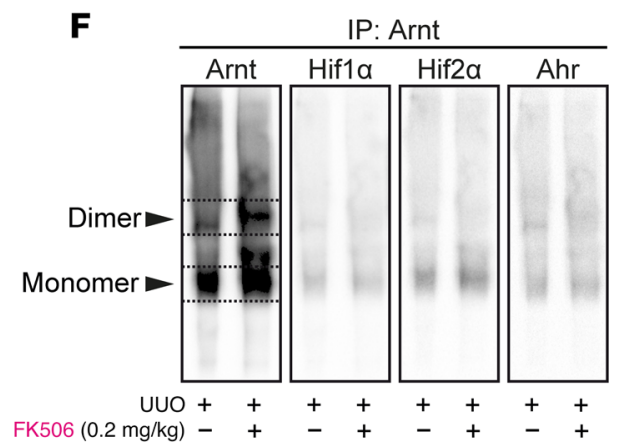

Figure 7. Evidence for Arnt homodimer formation in mice treated with FK506. (A and B) Representative kidney sections of $\gamma G T^{c r e+} ; Y y 7^{f l / f l}$ and $\gamma G T^{c r e-} ; Y y 7^{f l / f l}$ control mice immunolabeled with primary antibodies against Arnt are shown. $n=3 /$ group. Data are presented as mean $\pm \mathrm{SD}$. ${ }^{* *} P<0.01$; ${ }^{* * *} P<0.001$, 1-way ANOVA with Bonferroni's post hoc analysis. (C-E) Arnt protein levels were analyzed by immunoblotting and immunostaining. Scale bars: $25 \mu \mathrm{m}$. $n=$ 6/group. Data are presented as mean $\pm \mathrm{SD}$. ${ }^{* *} P<0.001$; ${ }^{*} P<0.0001,1$-way ANOVA with Bonferroni's post hoc analysis. (F) Dimer formation of Arnt/Arnt, Arnt/Hif1 $\alpha$, Arnt/Hif2 $\alpha$, and Arnt/Ahr in total kidney lysates was assessed by native gel electrophoresis after Arnt pulldown. See complete unedited blots in the supplemental material.

ings, FKBP12 and YY1 were equally detectable in human pathologies, including chronically injured kidneys, livers, and lungs, as prerequisites of identified organ protection mediated by FK506 (Supplemental Figure 10, A-C, and Supplemental Table 6). To elucidate whether modulation of an FKBP12/YY1/ARNT/ALK3 signaling axis was not only limited to mice, but was similarly effective in humans, we next exposed human TECs to FK506 and analyzed efficacy to induce protective ARNT. Exposure of human TEC cultures to previous established picomolar FK506 equally induced ARNT transcription and ALK3 (Figure 13, A and B). Because FK506 has been in clinical use for decades to prevent rejection of kidney transplants at high immunosuppressive doses (with well-established adverse toxicity likely masking beneficial effects) and not at the low doses at which we observed optimal renoprotection in rodents, we focused in our analysis on the induction of ARNT expression and subsequent ALK3-mediated
BMP-signaling responses in kidney allografts matched for comparable kidney function, chronic tubular injury, and extent of interstitial fibrosis (Supplemental Figure 11, A-F, and Supplemental Table 7). Immunolabeling confirmed the presence of FKBP12 and YY1 in all allografts (Figure 13C), while enhanced ARNT and $A L K 3$ transcription was present in kidney biopsies of patients on an FK506-based immunosuppressive regimen as compared with those receiving CsA (Figure 13, C-H).

\section{Discussion}

Here, we report a renoprotective mechanism that is controlled by the transcription factor ARNT, which effectively inhibits progression of chronic kidney injury by inducing $A L K 3$ transcription. We further report that ARNT expression itself is controlled by the FKBP12/YY1 transcriptional repressor complex (Supplemental Figure 12A) and that disruption of such FKBP12/YY1 complexes 
A
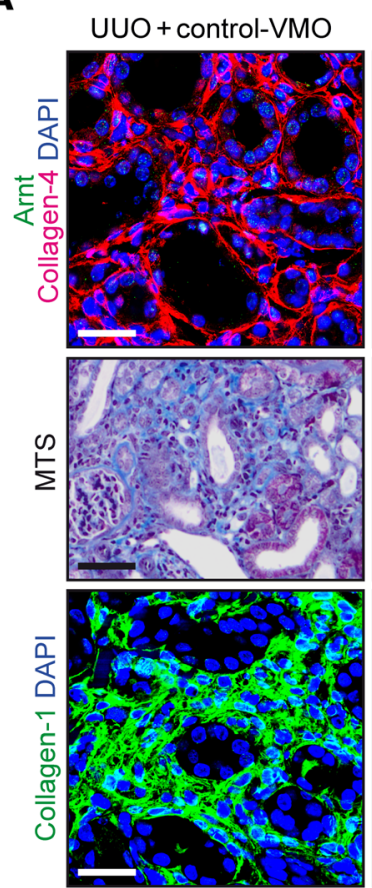

B

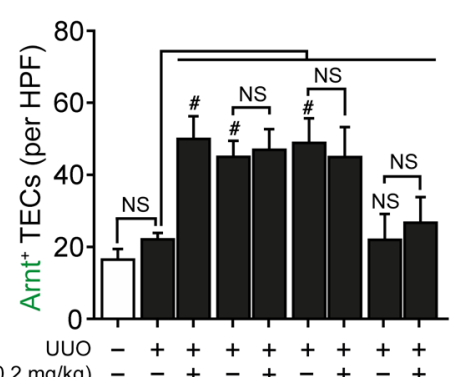

FK506 $(0.2 \mathrm{mg} / \mathrm{kg})-\mathbf{+}+\mathbf{+}+\mathbf{+}+$

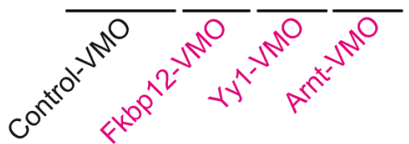

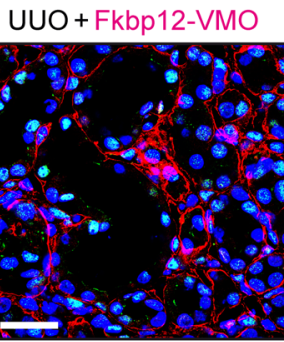
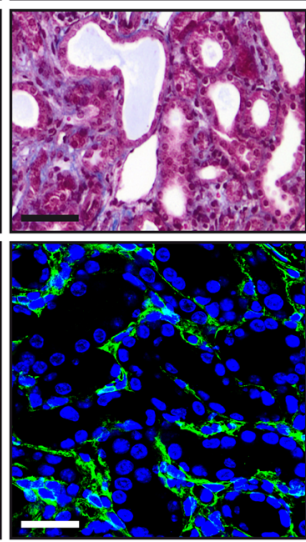

C
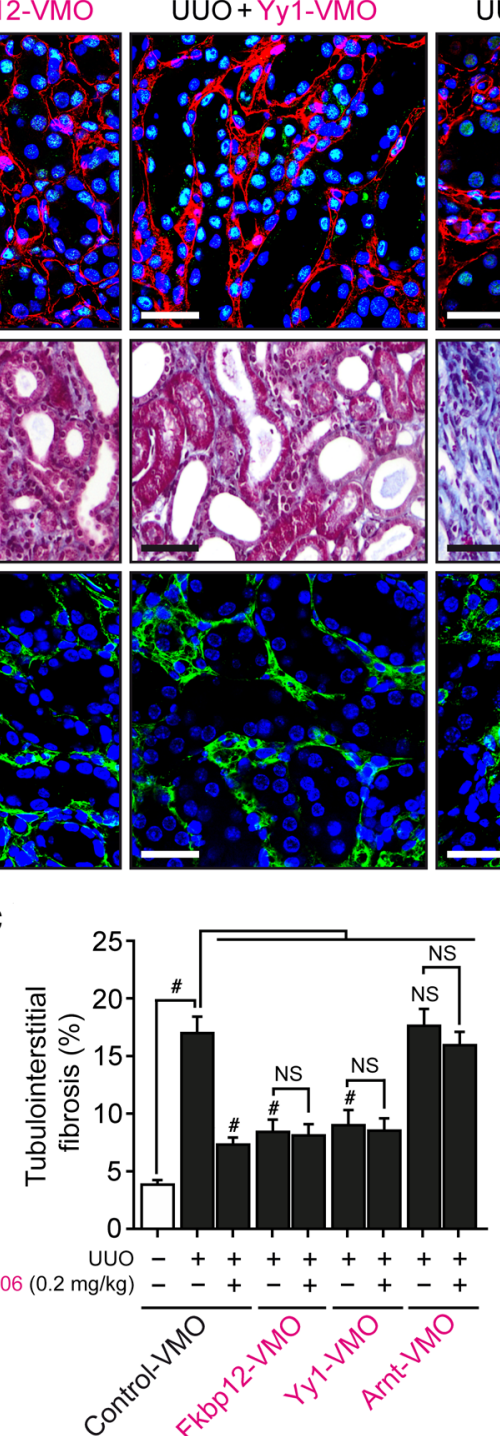

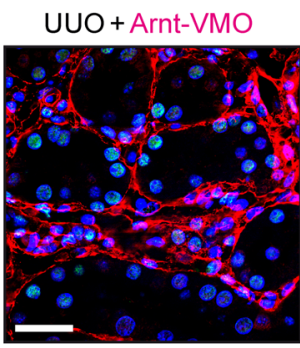

$\mathrm{UUO}+$ Arnt-VMO

+ FK506 $(0.2 \mathrm{mg} / \mathrm{kg})$
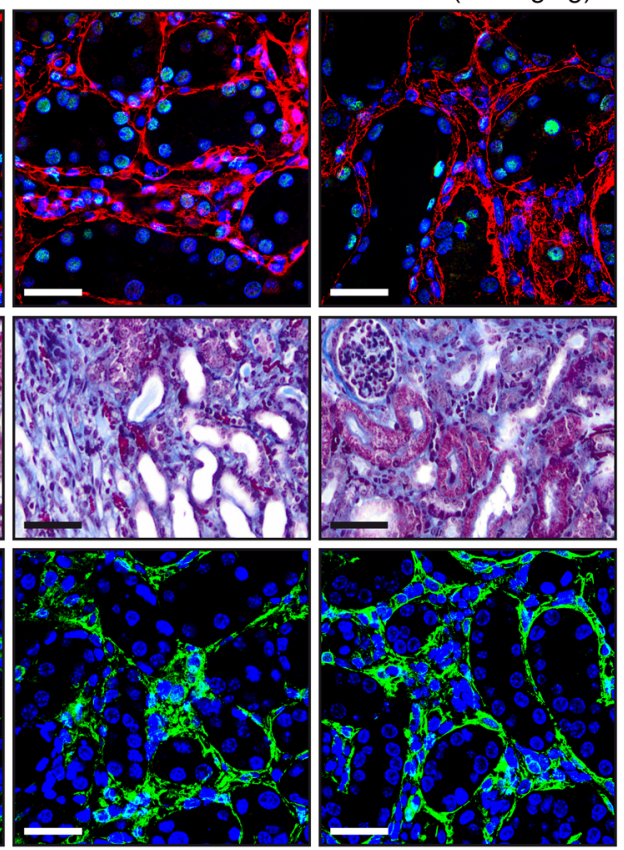

D

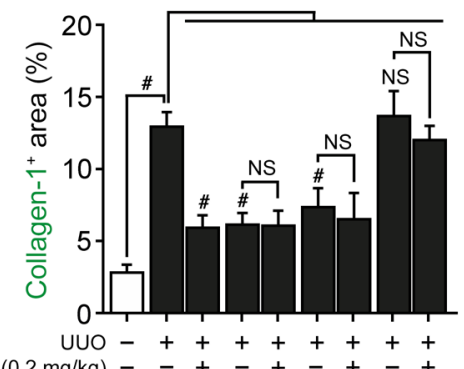

FK506 $(0.2 \mathrm{mg} / \mathrm{kg})--+-+-++$

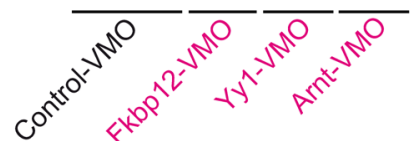

Figure 8. Selective targeting of FKBP12/YY1 effectively modulates protective ARNT within chronically injured kidneys. (A-D) Mice were treated daily with intraperitoneal administration of either control VMOs or VMOs targeting the translational start site of Fkbp12 (Fkbp12-VMO), Yy1 (Yy1-VMO), or Arnt (Arnt-VMO) starting 2 days prior to surgery and were orally treated with either vehicle buffer or FK506 (0.2 mg/kg orally per day) starting 1 day prior to surgery. Representative photomicrographs of kidney sections labeled for Arnt, MTS, and collagen-1 are shown. Scale bars: $25 \mu \mathrm{m}$ (Arnt, collagen-1); $50 \mu \mathrm{m}$ (MTS). $n=6$ /group. Data are presented as mean \pm SD. ${ }^{P} P<0.0001$, 1-way ANOVA with Bonferroni's post hoc analysis.

by picomolar FK506 at subimmunosuppressive doses, small molecule FKBP12 inhibitor GPI-1046, or direct targeting of FKBP12/ YY1 using VMOs increases ARNT levels (Supplemental Figure 12B). Subsequent activation of ALK3-dependent canonical BMPsignaling responses by ARNT homodimer formation (independently of HIF1 $\alpha$ or AHR) attenuates chronic organ failure in models of chronic kidney, cardiac, and liver injuries (Supplemental Figure 12, C and D). The identified FKBP12/YY1/ARNT/ALK3 signaling axis is supported by mining of public expression profiling databases in various organs: in the context of the kidney, an inverse correlation between intrarenal $A L K 3$ expression and FKBP12/YY1 was confirmed by several array data sets performed in different renal pathologies (Supplemental Figure 13, A-F) (39-44), confirmed in microdissected renal tubules (Supplemental Figure 14A) (45). Fur- thermore, kidney allografts of patients on an FK506-based immunosuppressive regimen displayed enhanced intrarenal $A R N T$ and $A L K 3$ transcription as compared with allografts of patients on CsA (Supplemental Figure 14, B and C) (44). Organ protection by the FKBP12/YY1/ARNT/ALK3 signaling axis is in line with extensive literature on 2 previously unconnected lines of research, as several studies have highlighted the efficacy of low-dose FK506 administration to protect against acute experimental injuries, including those of the kidney, heart, and liver (5-7), and multiple preclinical studies established beneficial effects of ALK3-mediated BMP signaling in these organs (19-23).

At the mechanistic level, we report that the homodimerization of ARNT is critical to eliciting its function in inducing ALK3 transcription. ARNT in general is a member of the PAS domain 
A

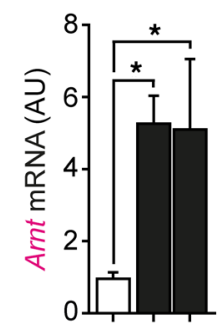

FK506 (200 pM) - + $\mathrm{GPI}-1046(10 \mathrm{uM})$ - - +
B

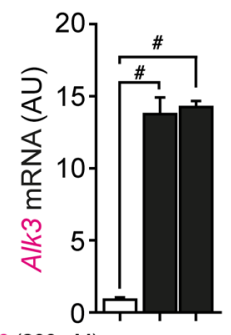

FK506 (200 pM) - + $\mathrm{GPI}-1046(10 \mu \mathrm{M})-$ +

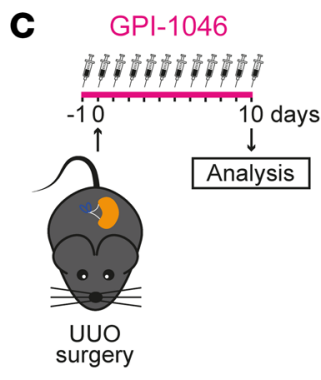

G

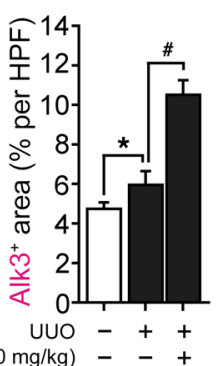

$\mathrm{GPI}-1046(10 \mathrm{mg} / \mathrm{kg})-+$

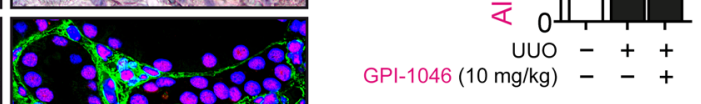

$\mathbf{J}$

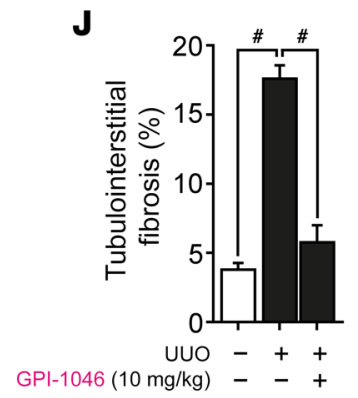

D

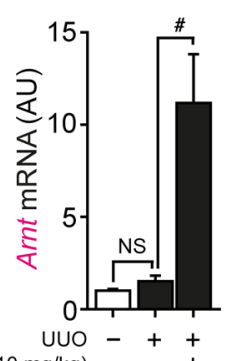

GPI-1046 (10 mg/kg) ++
$\mathbf{E}$

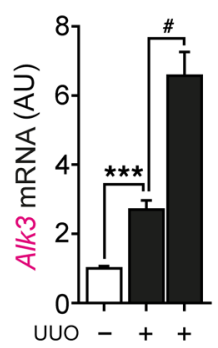

$\mathbf{F}$
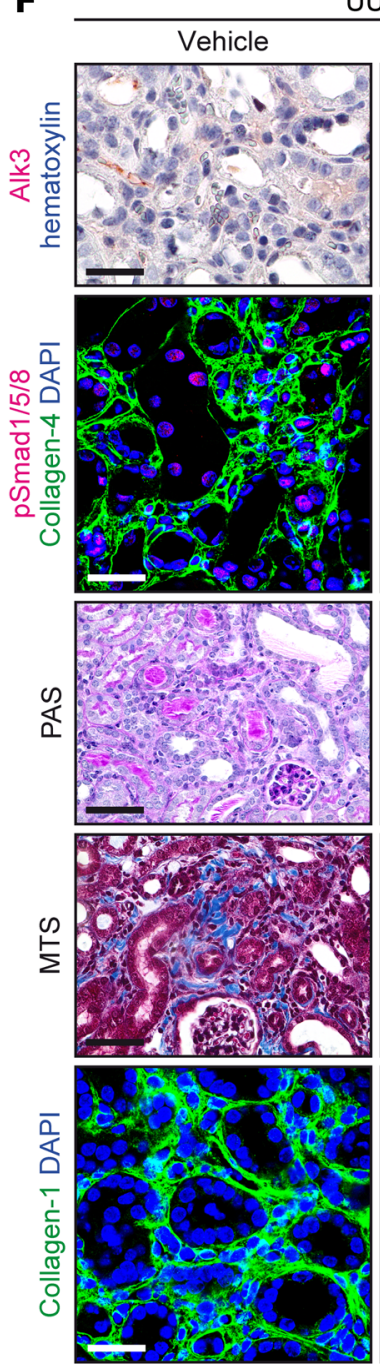
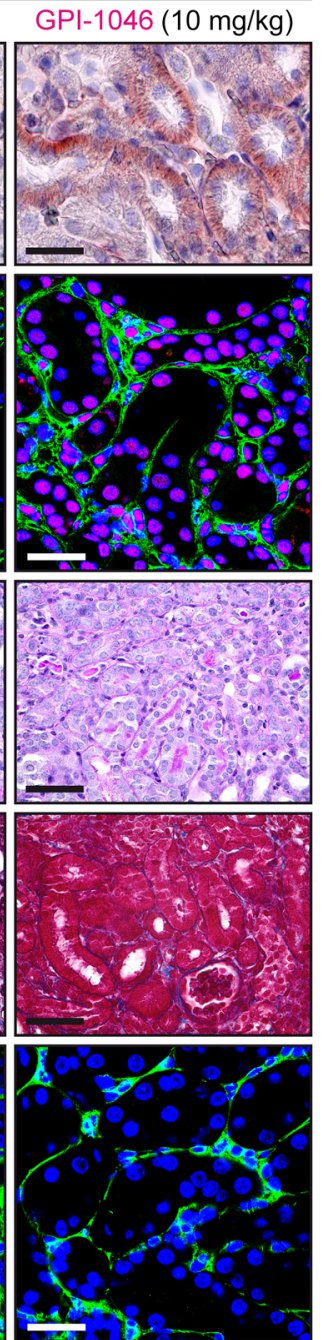

H

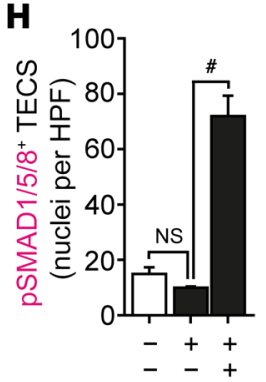

I

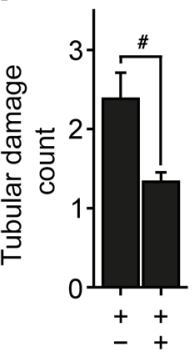

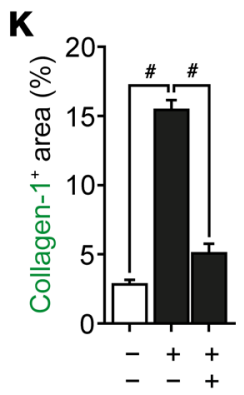

Figure 9. Selective targeting of FKBP12 by GPI-1046 effectively protects from chronic renal failure. (A and B) TECs were exposed to vehicle, FK506 (200 pM), or GPI-1046 (10 $\mu \mathrm{M})$, and relative Arnt and Alk3 mRNA expression levels were analyzed by qRT-PCR. $n=3$ independent experiments. Data are presented as mean $\pm \mathrm{SD}$. ${ }^{*} P<0.05$; ${ }^{*} P<0.0001$, 1-way ANOVA with Bonferroni's post hoc analysis. (C) Mice were challenged with UUO and treated with either vehicle buffer or GPI-1046 (10 mg/kg subcutaneously per day) starting 1 day prior to surgery. (D and E) Analysis was performed by qRT-PCR 10 days after UUO. Intrarenal Arnt and Alk3 mRNA expression levels are shown. $n=4$ /group. Data are presented as mean \pm SD. ${ }^{* *} P<0.001$; $P<0.0001,1$-way ANOVA with Bonferroni's post hoc analysis. (F-K) Representative photomicrographs of immunostaining for Alk3, p-Smad1/5/8, PAS-stained fibrotic kidney sections, MTS, and collagen-1 are shown. Scale bars: $25 \mu \mathrm{m}$ (Alk3, p-Smad1/5/8, collagen-1); $50 \mu \mathrm{m}$ (PAS, MTS). $n=6 /$ group. Data are presented as mean \pm SD. ${ }^{*} P<0.05,{ }^{*} P<0.0001,1$-way ANOVA with Bonferroni's post hoc analysis.

family, which is predominantly known to heterodimerize with other PAS family members to form heterodimeric transcription factors, classically with an $\alpha$ subunit of HIF or the dioxin receptor AHR to mediate hypoxia or xenobiotic responses by targeting genomic E-box motifs (32). A unique function of homodimeric ARNT as compared with heterodimeric ARNT is supported by recent studies that established the palindromic E-box motif CACGTG as a critical binding site for ARNT homodimers, while 
A

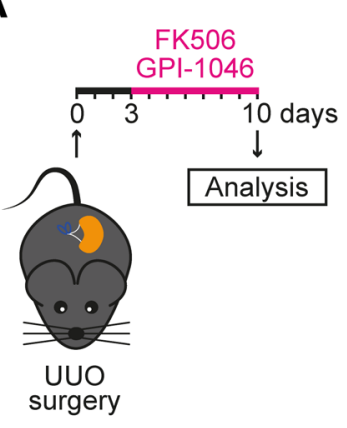

D
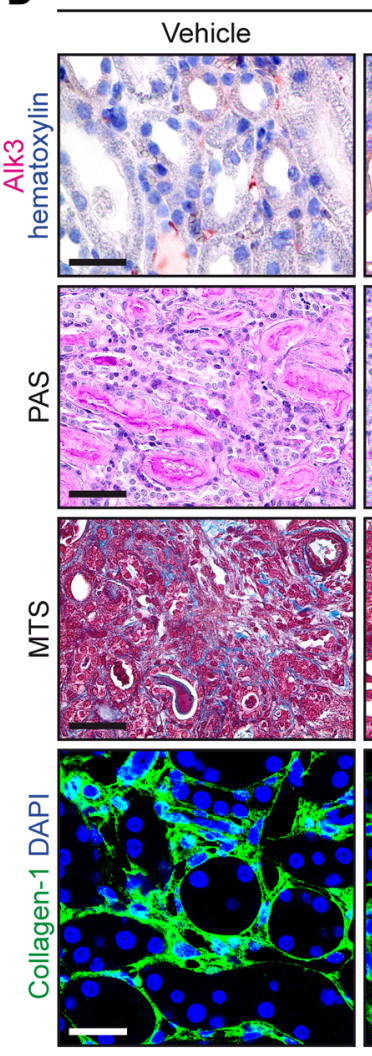

B

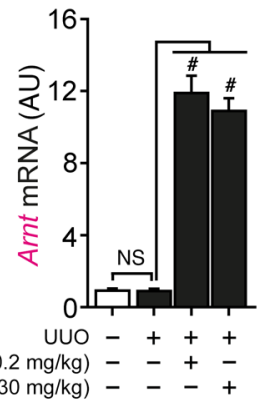

C

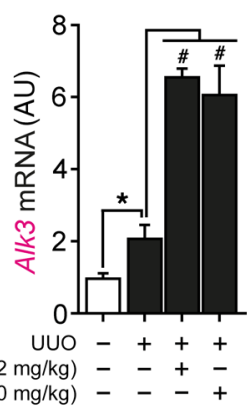

UUO
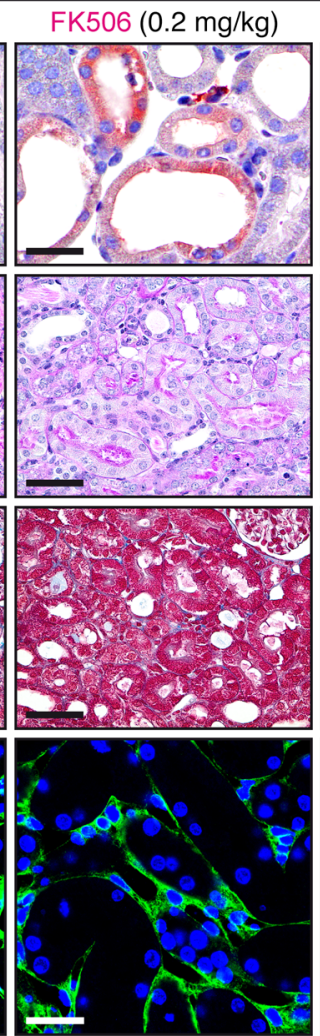
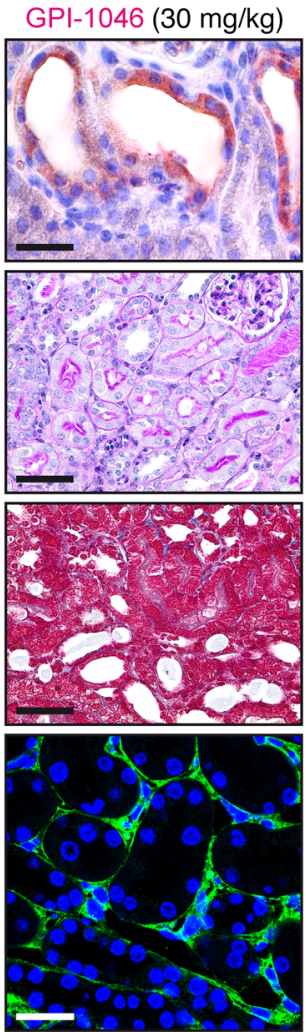

E

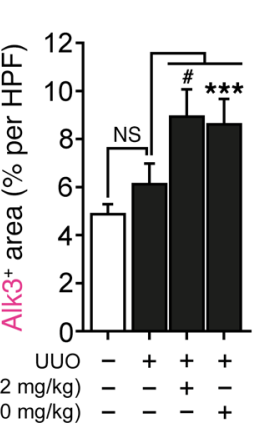

G

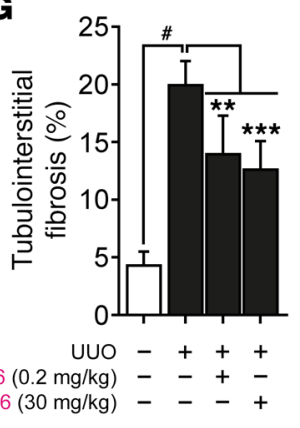

$\mathbf{F}$

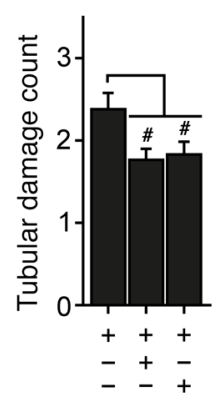

H

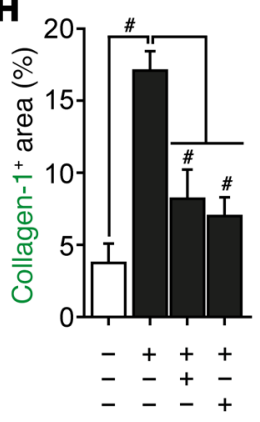

Figure 10. Selective targeting of FKBP12 by GPI-1046 protects from already established fibrotic lesions. (A) Mice were challenged with UUO and administered either vehicle buffer, FK506 (0.2 mg(kg orally per day), or GPI-1046 (30 mg/kg orally per day) starting 3 days after UUO surgery. (B and C) Analysis was performed by qRT-PCR. Intrarenal Arnt and Alk3 mRNA expression levels were assessed. $n=4$ /group. Data are presented as mean \pm SD. ${ }^{*} P<0.05$, ${ }^{\#} P<0.0001$, 1-way ANOVA with Bonferroni's post hoc analysis. (D-H) Representative photomicrographs of immunostainings for Alk3, PAS-stained fibrotic kidney sections, MTS, and collagen-1 are shown. Scale bars: $25 \mu \mathrm{m}$ (Alk3, collagen-1); $50 \mu \mathrm{m}$ (PAS, MTS). $n=6 /$ group. Data are presented as mean \pm SD. ${ }^{* *} P<0.01 ;{ }^{* *} P<0.001 ;{ }^{*} P<0.0001,1$-way ANOVA with Bonferroni's post hoc analysis.

ARNT heterodimers bind to asymmetric E-box motifs in hypoxic/ xenobiotic response elements $(33,34)$. As ARNT homodimerization potentially provides another therapeutic target, additional research is warranted to explore the underlying mechanisms. In this regard, our studies suggest that the fate decision of homodimerization over heterodimerization is in part regulated by intracellular ARNT levels controlled by FKBP12/YY1 transcriptional repressor complexes. This is supported by array data sets confirming transcriptional $A R N T$ induction when YY1 was depleted (Supplemental Figure 14, D and E) (46) and binding of YY1 repressor to the ARNT proximal promoter (47). In addition, clustering of transcriptome array data sets revealed inverse correlation between $A R N T$ and FKBP12/YY1 in multiple organ systems, including renal, cardiovascular, and digestive tissues, confirming our findings (GEO GSE3526, Supplemental Table 8) (48). In this context, previous reports implicate activation of a YY1 signaling axis as detectable in renal, cardiac, hepatic, and pulmonary pathologies and show that YY1 depletion protects from chronic organ failure (49-51).

It is tempting to speculate that modulation of the newly identified FKBP12/YY1/ARNT/ALK3 signaling axis may be a promising target in chronic failure of multiple organ systems. In this regard, low-dose FK506 has already entered clinical testing (ClinicalTrials.gov NCT01647945) and shown promise in 
A

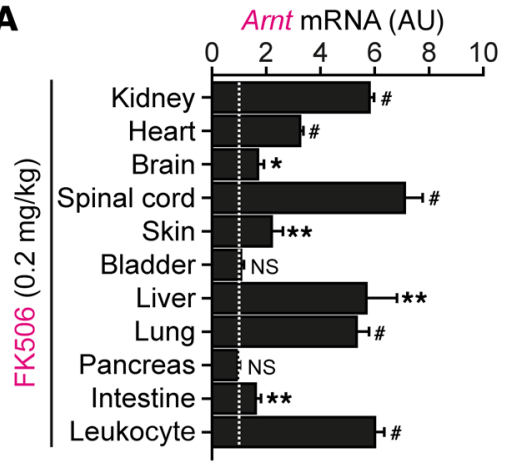

B

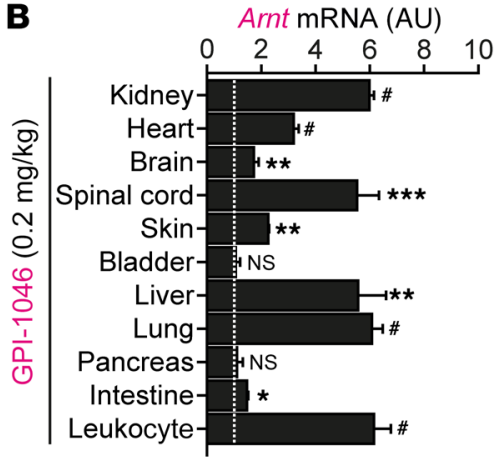

C

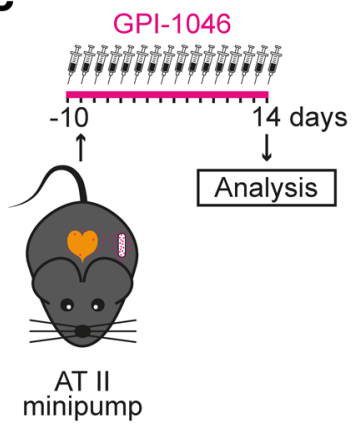

D

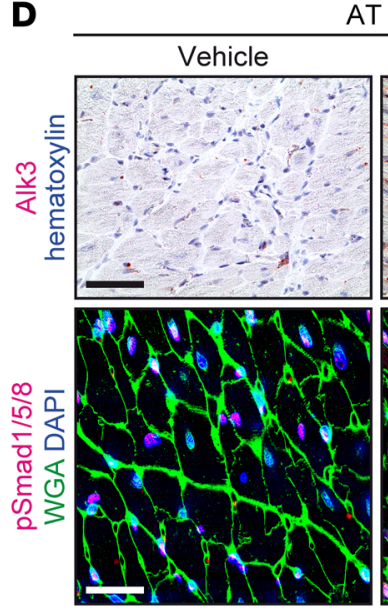

AT II
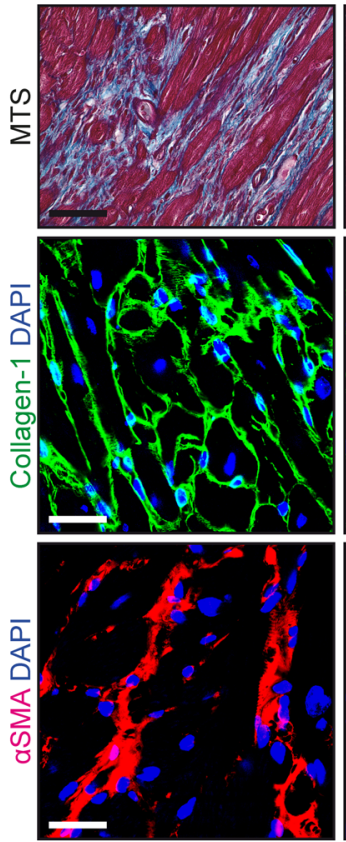

E

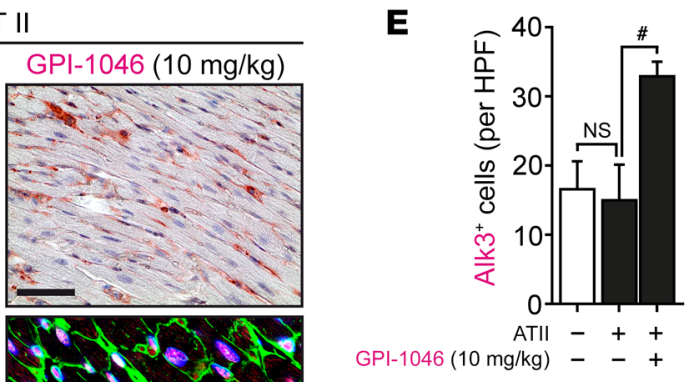

H

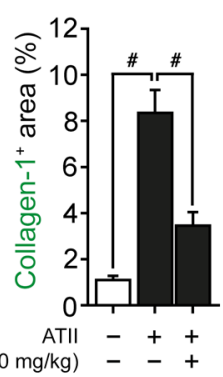

J
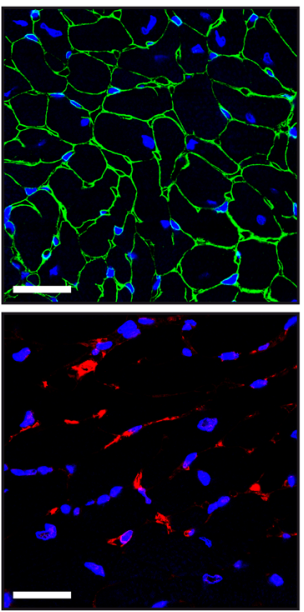

F
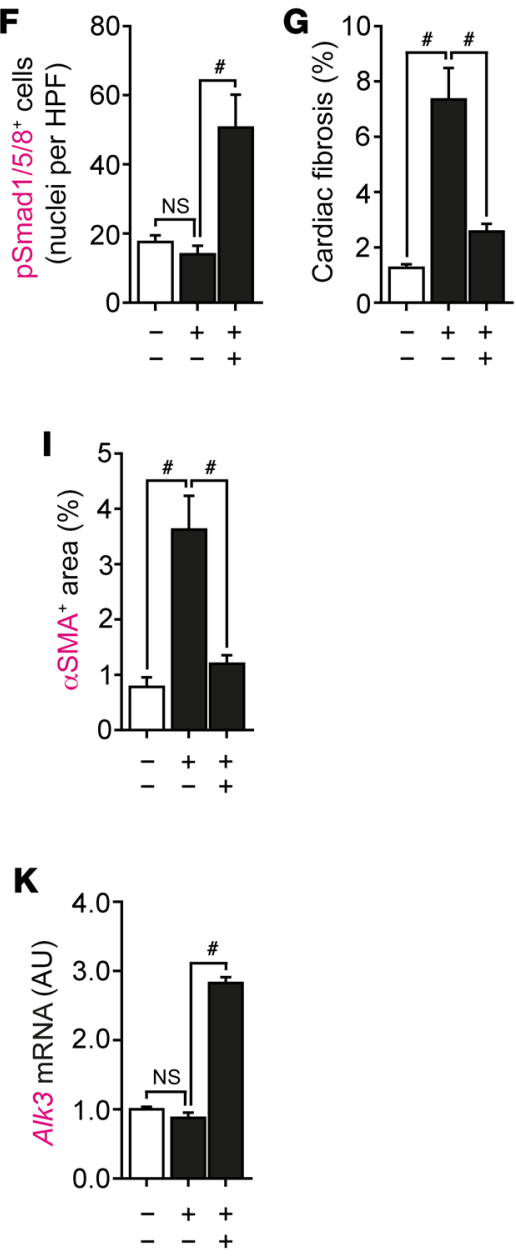

Figure 11. Pharmacological modulation of an FKBP12/YY1/ARNT/ALK3 signaling axis protects from chronic heart failure. (A and B) Analysis was performed by qRT-PCR. Arnt mRNA expression levels are shown in response to FK506 ( $0.2 \mathrm{mg} / \mathrm{kg}$ orally per day) or GPI-1046 (30 mg/kg orally per day). $n=3$ / group. Data are presented as mean $\pm \mathrm{SD}$. ${ }^{*} P<0.05$; ${ }^{* *} P<0.01$; ${ }^{* *} P<0.001 ;{ }^{*} P<0.0001$, 1-way ANOVA with Bonferroni's post hoc analysis in comparison with vehicle-treated control mice. (C) Mice were challenged with AT II delivered by osmotic minipumps. Vehicle buffer or GPI-1046 (10 mg/kg subcutaneously per day) was administered starting 1 day prior to AT II administration. (D-I) Representative photomicrographs of immunostainings for Alk3, p-Smad1/5/8, MTS, collagen-1, and $\alpha$ SMA in mice challenged with AT II are shown. Scale bars: $25 \mu \mathrm{m}$ (Alk3, p-Smad1/5/8, collagen-1, $\alpha$ SMA); $50 \mu \mathrm{m}$ (MTS). $n=6$ /group. Data are presented as mean \pm SD. ${ }^{P} P<0.0001$, 1-way ANOVA with Bonferroni's post hoc analysis. (J and $\left.\mathbf{K}\right)$ Arnt and Alk3 mRNA expression levels were analyzed by qRT-PCR. $n=4$ /group. Data are presented as mean \pm SD. ${ }^{\#} P<0.0001,1$-way ANOVA with Bonferroni's post hoc analysis. 
A

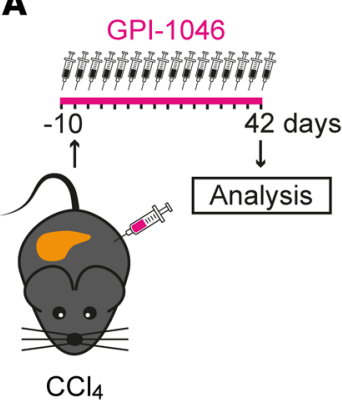

B

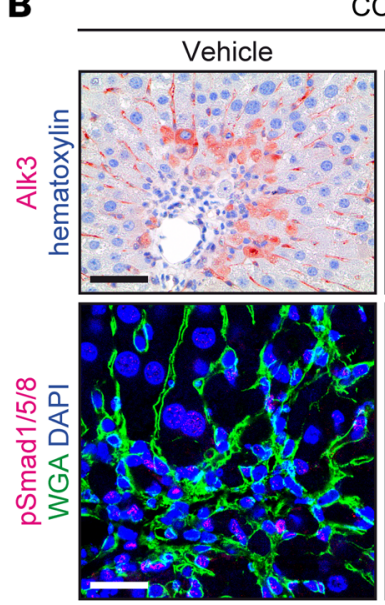

$\mathrm{CCl}_{4}$

GPI-1046 $(10 \mathrm{mg} / \mathrm{kg})$
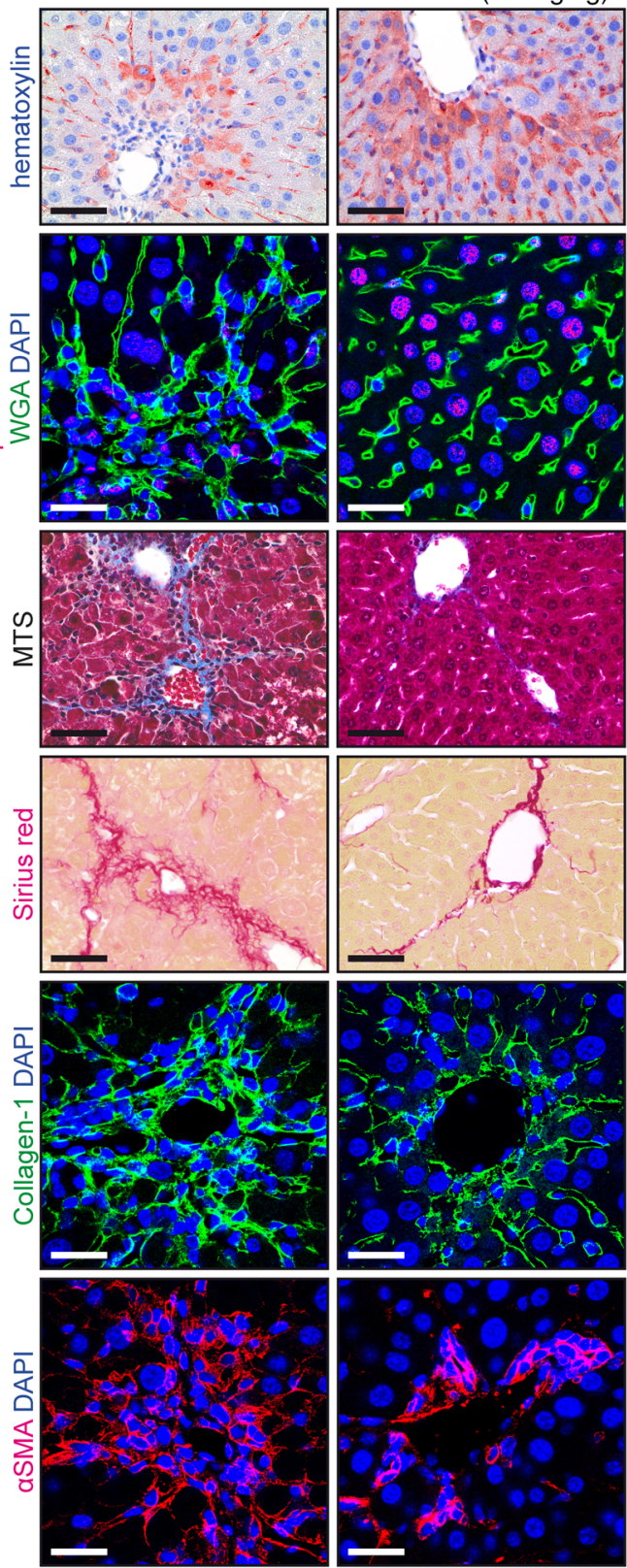

C
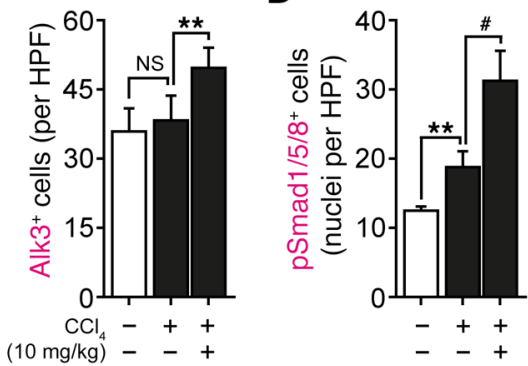

E

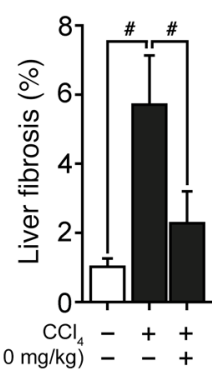

G

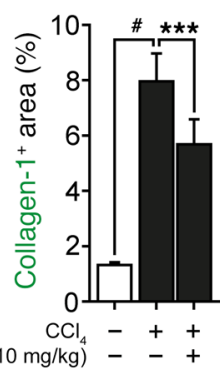

$\mathbf{F}$

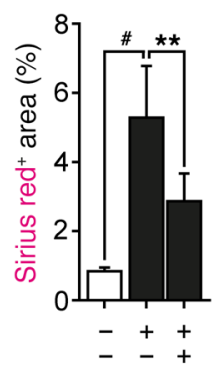

H

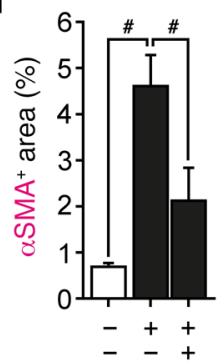

Figure 12. Pharmacological modulation of an FKBP12/YY1/ARNT/ALK3 signaling axis protects from chronic liver failure. (A) Mice were challenged with intraperitoneal injections of $\mathrm{CCl}_{4}$. Vehicle buffer or $\mathrm{CPI}-1046(10 \mathrm{mg} / \mathrm{kg}$ subcutaneously per day) was administered starting at 1 day prior to starting CCL4 injections. (B-H) Representative photomicrographs of immunostainings for Alk3, p-Smad1/5/8, MTS-stained fibrotic kidney sections, Sirius red, collagen-1, and $\alpha$ SMA in mice challenged with $\mathrm{CCl}_{4}$ are shown. Scale bars: $25 \mu \mathrm{m}$ (Alk3, p-Smad1/5/8, collagen-1, $\alpha \mathrm{SMA}$ ); $50 \mu \mathrm{m}$ (MTS, Sirius red). $n=5-7 /$ group Data are presented as mean $\pm \mathrm{SD}$. ${ }^{* *} P<0.01 ;{ }^{* *} P<0.001 ;{ }^{*} P<0.0001,1$-way ANOVA with Bonferroni's post hoc analysis.

pulmonary arterial hypertension patients in inducing protective BMP-signaling responses (52). Observed beneficial efficacy of FK506 was counterintuitive at first sight because decades of use as an immunosuppressant upon kidney transplantation had revealed its CNI nephrotoxicity, thereby limiting its clinical use (14). However, our studies established that FK506-induced organ protection is independent of CNI activity, as it is achieved at picomolar doses far below the nanomolar immunosuppressive regimens. Hence, we provide mechanistic evidence for why protective activity of FK506 in interventional therapeutic regimens has remained elusive thus far. Efficacy of the specific FKBP12 inhibitor GPI-1046 in protecting and attenuating disease progression in kidney, heart, and liver validated observed efficacy of FK506 and also provided a more specific tool from a translational perspective. Thus, we speculate that pharmacologic modulation of ARNT homodimerization and FKBP12/YY1 complexes may have translational potential for organ protection in the future. 
A

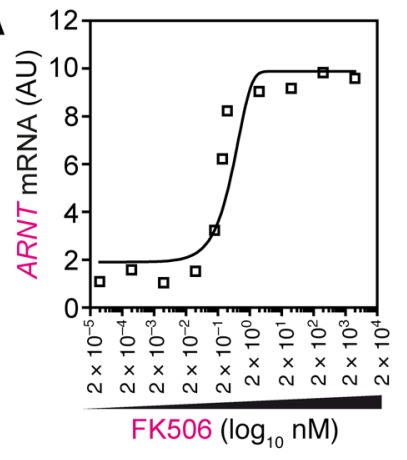

C
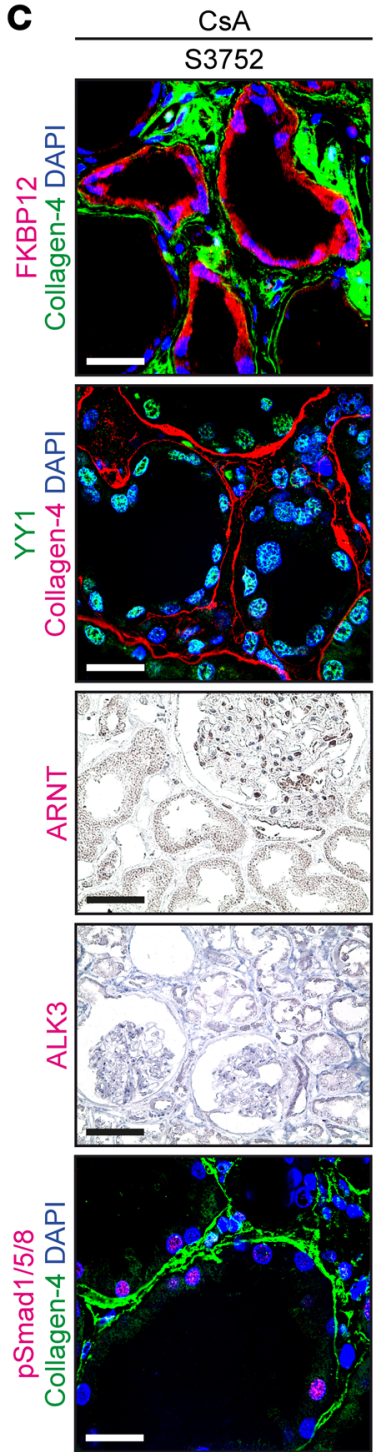

B

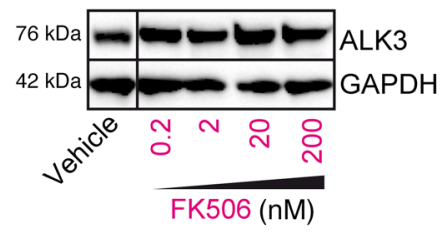

D

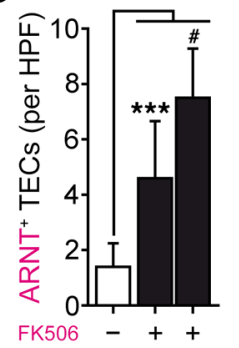

$5^{34^{2} c^{6^{4}} 5^{3} 0^{60}}$
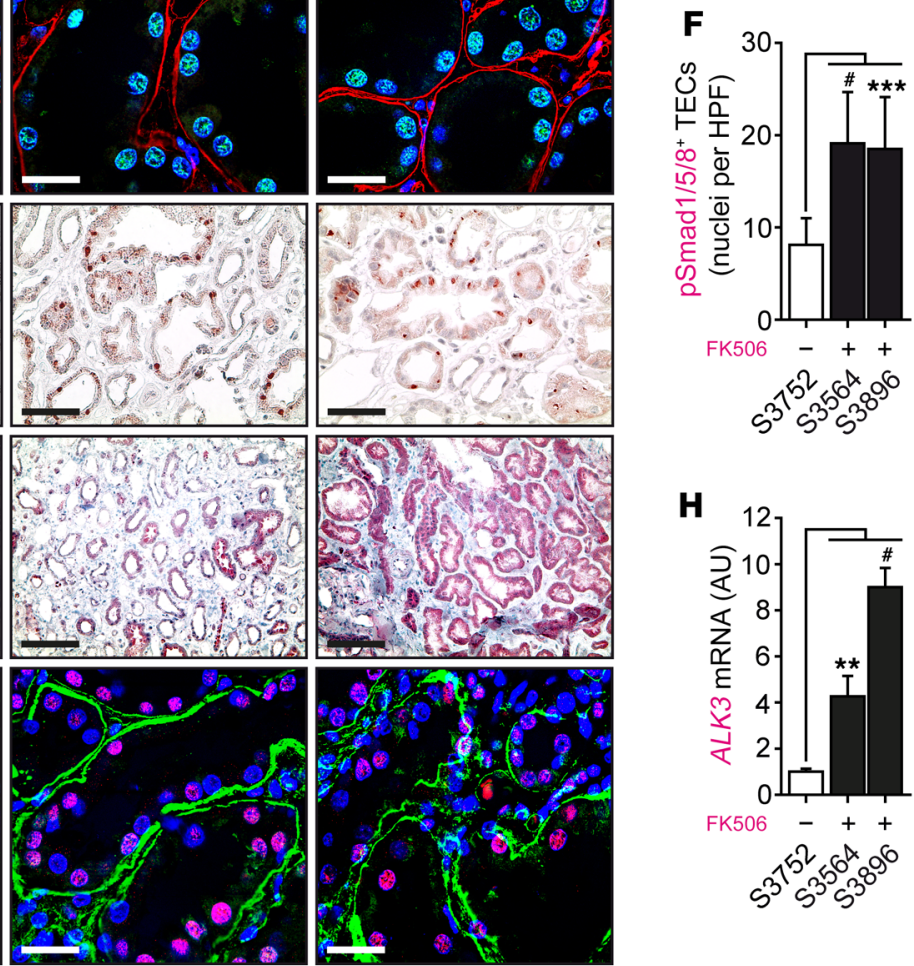

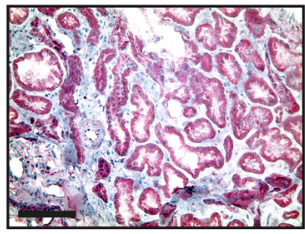

FK506
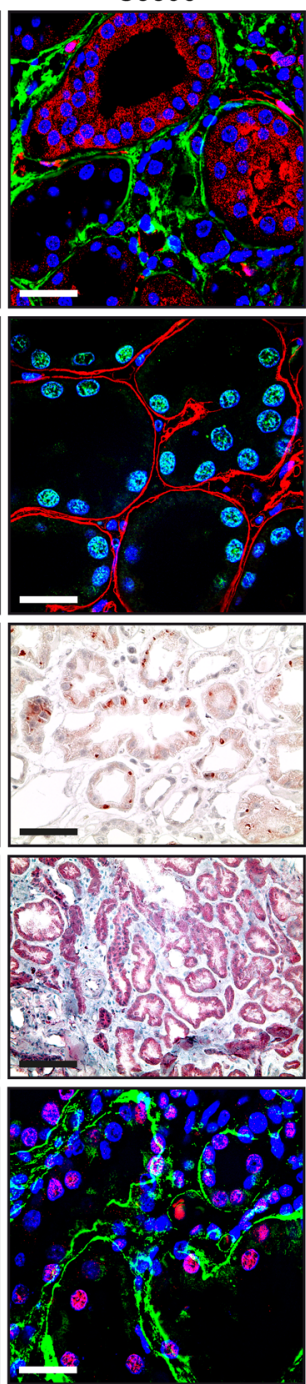

H

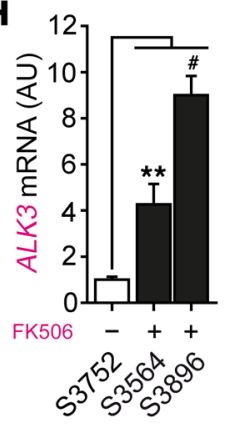

E

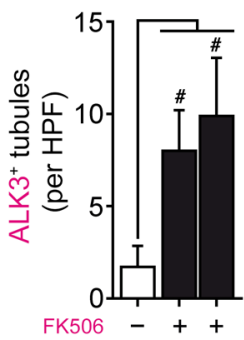

$10^{2}, 6^{x}+0^{\circ}$

$\mathrm{s}^{3} \mathrm{c}^{3} \mathrm{c}^{30}$

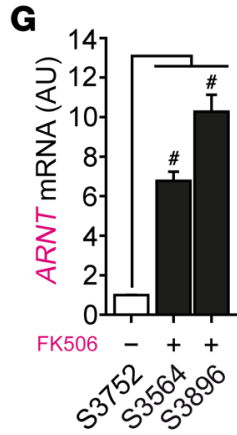

Figure 13. An FKBP12/YY1/ARNT signaling axis translates to humans. (A) Human TEC cultures were exposed to vehicle or indicated concentrations of FK506. ARNT mRNA expression was analyzed by qRT-PCR ( $n=3$ independent experiments). (B) Analysis was performed by SDS-PACE and subsequent immunoblotting. ALK3 was assessed in response to FK506. See complete unedited blots in the supplemental material. (C-H) In a small cohort of kidney transplant recipients with comparable histological patterns and immunosuppressive regimens based on CsA or FK506, kidney sections were immunolabeled with the following primary antibodies: FKBP12, YY1, ARNT, ALK3, and p-Smad1/5/8. Scale bars: $25 \mu \mathrm{m}$ (FKBP12, YY1, p-Smad1/5/8); 50 $\mu \mathrm{m}$ (ARNT); $100 \mu \mathrm{m}$ (ALK3). Measurements were done in 10 visual fields. Data are presented as mean \pm SD. ${ }^{* *} P<0.001$; ${ }^{*} P<0.0001,1$-way ANOVA with Bonferroni's post hoc analysis. (G and $\mathbf{H}) A R N T$ and $A L K 3$ mRNA expression levels were assessed by qRT-PCR. Measurements were done in technical triplicates. Data are presented as mean $\pm \mathrm{SD}$. ${ }^{* *} P<0.01 ;{ }^{\#} P<0.0001,1$-way ANOVA with Bonferroni's post hoc analysis. 


\section{Methods}

Generation of $\gamma G T^{\text {ret }} ; Y y 1^{f l / f l}$ mice. B6;129S4-Yy $1^{\text {tm2Yshi }} / J$ (referred as $Y y 1^{f / f l}$ ) mice were obtained from the Jackson Laboratory, $\mathrm{Tg}(\mathrm{Ggt1}$-cre $) \mathrm{M} 3 \mathrm{Egn} / \mathrm{J}$ (referred as $\gamma G T^{\text {rret }}$ ) mice were previously described, and genetic backgrounds were identical when comparing experimental groups (19).

UUO. Eight- to twelve-week-old C57BL/6, $\gamma G T^{\text {ret }} ; Y y 1^{f / f l}$, and $\gamma G T^{\text {tre- }} ; Y y 1^{f l / f l}$ control mice were anesthetized with isoflurane inhalation (2\%-3\%), and analgesia was performed by subcutaneous injection of $0.1 \mathrm{mg} / \mathrm{kg}$ body weight per day of buprenorphine. The ureter was separated from the surrounding tissues, and 2 ligatures were placed about $5 \mathrm{~mm}$ apart in the upper two-thirds of the left ureter to obtain reliable obstruction. Mice were sacrificed 3, 7, or 10 days after UUO for further analyses, as described previously (53).

AT II-induced cardiac hypertrophy and fibrosis. Eight- to twelveweek-old $C 57 \mathrm{BL} / 6$ mice were anesthetized with isoflurane inhalation (2\%-3\%), and analgesia was performed by subcutaneous injection of $0.1 \mathrm{mg} / \mathrm{kg}$ body weight of buprenorphine per day. Osmotic minipumps (Alzet) were loaded with AT II to continuously deliver $1.44 \mu \mathrm{g} /$ $\mathrm{kg}$ body weight per day and implanted subcutaneously (38). Measurements of blood pressure were performed using a tail-cuff system. Systolic, diastolic, and mean arterial pressure (MAP) as well as heart rate were recorded, and mice were sacrificed 14 days after implantation for further analyses.

$\mathrm{CCl}_{4}$-induced liver fibrosis. Eight- to twelve-week-old C57BL/6 mice were intraperitoneally injected with 0.25 (first injection), 0.5 (second injection), and $1 \mathrm{ml} / \mathrm{kg}$ body weight $\mathrm{CCl}_{4}(25 \% \mathrm{v} / \mathrm{v}$ dissolved in sterile oil) twice a week (23). Mice were sacrificed after 42 days for further analyses.

Cell culture. HK-2 cells (ATTC) are immortalized proximal tubule epithelial cells derived from normal adult human kidney. The murine proximal TEC line MCT was generated from the renal cortex of SJL mice (54). Routine testing confirmed that all cell lines were negative for the presence of mycoplasma contamination. Cell lines used in this manuscript are not listed in the International Cell Line Authentication Committee (ICLAC) Register of Misidentified Cell Lines (http:// iclac.org/databases/cross-contaminations/) or the NCBI BioSample database (https://www.ncbi.nlm.nih.gov/biosample) of misidentified cell lines. Cells were cultured in DMEM (Gibco, Thermo Fisher Scientific) medium supplemented with $100 \mathrm{~g} / \mathrm{ml}$ penicillin, $100 \mathrm{~g} /$ $\mathrm{ml}$ streptomycin, and 10\% heat-inactivated FBS (MilliporeSigma) at $37^{\circ} \mathrm{C}$ in $5 \% \mathrm{CO}_{2}$.

Further material and methods details are listed in Supplemental Experimental Procedures.
Statistics. The numbers of individual mice and independent experiments are indicated in the corresponding figure legends. For single-group comparison, 2-tailed Student's $t$ test was used to determine statistical significance. One-way ANOVA with Bonferroni's post hoc analysis was used for multiple comparisons of samples to determine statistical significance. Linear regression was performed comparing indicated pairs of parameters, and $r^{2}$ and $P$ values are indicated in the corresponding graphs. Statistical significance was calculated with Prism 5 software (GraphPad) and defined as $P<0.05$.

Study approval. All animal studies were carried out with the approval of the Landesamt für Verbraucherschutz und Lebensmittelsicherheit (LAVES, Oldenburg, Germany) and the University Medical Center Göttingen. The use of parts of human specimens for research purposes was approved by the Ethics Committee of the University Medical Center Göttingen. All patients gave informed consent, and all samples were deidentified. A pathologist assessed for the presence of disease. Clinical data are presented in Supplemental Tables 6 and 7.

\section{Author contributions}

BT and DT performed, designed, and supervised experiments; collected and analyzed data; generated the figures; and cowrote the manuscript. GN, FK, GR, AK, and TL performed experiments and collected data. SH and GAM provided human specimens. MZ conceived the project, designed and supervised experiments, analyzed data, and cowrote the manuscript. EMZ, GAM, and RK edited the manuscript.

\section{Acknowledgments}

The authors thank Anika Krüger, Sarah Rinkleff, and Annika Faust for technical assistance. MZ was supported by the Deutsche Forschungsgemeinschaft (DFG-ZE523/3-1, ZE523/4-1, and SFB1002). EMZ was supported by DFG grant SFB1002. The work was supported by equipment grant INST 1525/16-1. BT and DT were supported by the Seed Funding Research Program of the Faculty of Medicine, Georg August University Göttingen (1402720 to BT and 1402910 to DT). Funding sources had no involvement in study design, data collection, analysis, and interpretation, writing, or decision to submit the article.

Address correspondence to: Michael Zeisberg, Clinic for Nephrology and Rheumatology, University Medicine Göttingen, Georg August University, Robert Koch Street 40, Göttingen, Germany. Phone: 49.551.39.20076; Email: mzeisberg@med.uni-goettingen.de.
1. Kapitsinou PP, Haase VH. Molecular mechanisms of ischemic preconditioning in the kidney. Am J Physiol Renal Physiol. 2015;309(10):F821-F834.

2. Joo JD, Kim M, D’Agati VD, Lee HT. Ischemic preconditioning provides both acute and delayed protection against renal ischemia and reperfusion injury in mice. J Am Soc Nephrol. 2006;17(11):3115-3123.

3. Murry CE, Jennings RB, Reimer KA. Preconditioning with ischemia: a delay of lethal cell injury in ischemic myocardium. Circulation. 1986;74(5):1124-1136.

4. Stringa $P$, et al. Pretreatment combination reduces remote organ damage secondary to intestinal reperfusion injury in mice: follow-up study. Transplant Proc. 2016;48(1):210-216.

5. Sakr M, et al. The protective effect of FK506 pretreatment against renal ischemia/ reperfusion injury in rats. Transplantation. 1992;53(5):987-991.

6. Feng X, et al. Protective effect of FK506 on myocardial ischemia/reperfusion injury by suppression of CaN and ASK1 signaling circuitry. Cardiovasc Toxicol. 2011;11(1):18-27.

7. Hüser N, et al. Graft preconditioning with lowdose tacrolimus (FK506) and nitric oxide inhibitor aminoguanidine (AGH) reduces ischemia/ reperfusion injury after liver transplantation in the rat. Arch Pharm Res. 2009;32(2):215-220.

8. Spiekerkoetter E, et al. FK506 activates BMPR2, rescues endothelial dysfunction, and reverses pulmonary hypertension. J Clin Invest. 2013;123(8):3600-3613.

9. Sharkey J, Butcher SP. Immunophilins mediate the neuroprotective effects of FK506 in focal cerebral ischaemia. Nature. 1994;371(6495):336-339.

10. Pan F, et al. [Protective effect of tacrolimus postconditioning on oxidative stress injury provoked by spinal cord ischemia-reperfusion in rats]. Zhonghua Yi Xue Za Zhi. 2013;93(47):3796-3800.

11. Nezami BG, et al. Pharmacological preconditioning of random-pattern skin flaps with local 
FK506 in nicotine-treated rats: interaction with nitric oxide system. J Plast Reconstr Aesthet Surg. 2009;62(12):e643-e644

12. Stringa $P$, et al. Ischemic preconditioning and tacrolimus pretreatment as strategies to attenuate intestinal ischemia-reperfusion injury in mice. Transplant Proc. 2013;45(6):2480-2485.

13. Schreiber SL, Crabtree GR. The mechanism of action of cyclosporin A and FK506. Immunol Today. 1992;13(4):136-142.

14. Ekberg H, et al. Reduced exposure to calcineurin inhibitors in renal transplantation. $N$ Engl J Med. 2007;357(25):2562-2575.

15. Jiang H, Yang X, Soriano RN, Fujimura T, Krishnan K, Kobayashi M. Distinct patterns of cytokine gene suppression by the equivalent effective doses of cyclosporine and tacrolimus in rat heart allografts. Immunobiology. 2000;202(3):280-292.

16. Karamperis N, Povlsen JV, Højskov C, Poulsen JH, Pedersen AR, Jørgensen KA. Comparison of the pharmacokinetics of tacrolimus and cyclosporine at equivalent molecular doses. Transplant Proc. 2003;35(4):1314-1318.

17. Lamb J, et al. The Connectivity Map: using gene-expression signatures to connect small molecules, genes, and disease. Science. 2006;313(5795):1929-1935.

18. Hieronymus $\mathrm{H}$, et al. Gene expression signaturebased chemical genomic prediction identifies a novel class of HSP90 pathway modulators. Cancer Cell. 2006;10(4):321-330.

19. Sugimoto H, et al. Activin-like kinase 3 is important for kidney regeneration and reversal of fibrosis. Nat Med. 2012;18(3):396-404.

20. Zeisberg M, et al. BMP-7 counteracts TGF-beta1induced epithelial-to-mesenchymal transition and reverses chronic renal injury. Nat Med. 2003;9(7):964-968.

21. Zeisberg M, et al. Bone morphogenic protein-7 inhibits progression of chronic renal fibrosis associated with two genetic mouse models. Am J Physiol Renal Physiol. 2003;285(6):F1060-F1067.

22. Zeisberg EM, et al. Endothelial-to-mesenchymal transition contributes to cardiac fibrosis. Nat Med. 2007;13(8):952-961.

23. Zeisberg M, et al. Fibroblasts derive from hepatocytes in liver fibrosis via epithelial to mesenchymal transition. J Biol Chem. 2007;282(32):23337-23347.

24. Yang G, Zhu Z, Wang Y, Gao A, Niu P, Tian L. Bone morphogenetic protein-7 inhibits silicainduced pulmonary fibrosis in rats. Toxicol Lett. 2013;220(2):103-108.

25. Chang CF, et al. Intravenous administration of bone morphogenetic protein-7 after ischemia improves motor function in stroke rats. Stroke.
2003;34(2):558-564.

26. Flier SN, Tanjore H, Kokkotou EG, Sugimoto H, Zeisberg M, Kalluri R. Identification of epithelial to mesenchymal transition as a novel source of fibroblasts in intestinal fibrosis. J Biol Chem. 2010;285(26):20202-20212.

27. Cuny GD, et al. Structure-activity relationship study of bone morphogenetic protein (BMP) signaling inhibitors. Bioorg Med Chem Lett. 2008;18(15):4388-4392.

28. Roider HG, Kanhere A, Manke T, Vingron M. Predicting transcription factor affinities to DNA from a biophysical model. Bioinformatics. 2007;23(2):134-141.

29. Szklarczyk D, et al. STRING v10: protein-protein interaction networks, integrated over the tree of life. Nucleic Acids Res. 2015;43(Database issue):D447-D452.

30. Galvin KM, Shi Y. Multiple mechanisms of transcriptional repression by YY1. Mol Cell Biol. 1997;17(7):3723-3732.

31. Heinemeyer T, et al. Databases on transcriptional regulation: TRANSFAC, TRRD and COMPEL. Nucleic Acids Res. 1998;26(1):362-367.

32. Antonsson C, Arulampalam V, Whitelaw ML, Pettersson S, Poellinger L. Constitutive function of the basic helix-loop-helix/PAS factor Arnt. Regulation of target promoters via the $\mathrm{E}$ box motif. J Biol Chem. 1995;270(23):13968-13972.

33. Swanson HI, Chan WK, Bradfield CA. DNA binding specificities and pairing rules of the $\mathrm{Ah}$ receptor, ARNT, and SIM proteins. J Biol Chem. 1995;270(44):26292-26302.

34. Sogawa K, et al. Possible function of Ah receptor nuclear translocator (Arnt) homodimer in transcriptional regulation. Proc Natl Acad Sci US A 1995;92(6):1936-1940.

35. Blum M, De Robertis EM, Wallingford JB, Niehrs C. Morpholinos: Antisense and Sensibility. Dev Cell. 2015;35(2):145-149.

36. Grande MT, et al. Snail1-induced partial epithelial-to-mesenchymal transition drives renal fibrosis in mice and can be targeted to reverse established disease. Nat Med. 2015;21(9):989-997.

37. Steiner JP, et al. Neurotrophic immunophilin ligands stimulate structural and functional recovery in neurodegenerative animal models. Proc Natl Acad Sci U S A. 1997;94(5):2019-2024.

38. Matsumoto E, et al. Angiotensin II-induced cardiac hypertrophy and fibrosis are promoted in mice lacking Fgf16. Genes Cells. 2013;18(7):544-553.

39. Ju W, et al. Tissue transcriptome-driven identification of epidermal growth factor as a chronic kidney disease biomarker. Sci Transl Med. 2015;7(316):316ra193.

40. Nakagawa S, et al. Molecular markers of tubu- lointerstitial fibrosis and tubular cell damage in patients with chronic kidney disease. PLOS ONE. 2015;10(8):e0136994.

41. Reich HN, et al. A molecular signature of proteinuria in glomerulonephritis. PLOS ONE. 2010;5(10):e13451.

42. Woroniecka KI, Park AS, Mohtat D, Thomas DB, Pullman JM, Susztak K. Transcriptome analysis of human diabetic kidney disease. Diabetes. 2011;60(9):2354-2369.

43. Schmid H, et al. Modular activation of nuclear factor-kappaB transcriptional programs in human diabetic nephropathy. Diabetes. 2006;55(11):2993-3003.

44. Flechner SM, et al. Kidney transplant rejection and tissue injury by gene profiling of biopsies and peripheral blood lymphocytes. Am J Transplant. 2004;4(9):1475-1489.

45. Ko YA, et al. Cytosine methylation changes in enhancer regions of core pro-fibrotic genes characterize kidney fibrosis development. Genome Biol. 2013;14(10):R108

46. Chen L, Shioda T, Coser KR, Lynch MC, Yang C, Schmidt EV. Genome-wide analysis of YY2 versus YY1 target genes. Nucleic Acids Res. 2010;38(12):4011-4026.

47. Guo AM, Sun K, Su X, Wang H, Sun H. YY1TargetDB: an integral information resource for Yin Yang 1 target loci. Database (Oxford). 2013;2013:bat007.

48. Roth RB, et al. Gene expression analyses reveal molecular relationships among 20 regions of the human CNS. Neurogenetics. 2006;7(2):67-80.

49. Liang S, et al. Novel mechanism of regulation of fibrosis in kidney tumor with tuberous sclerosis. Mol Cancer. 2013;12:49.

50. Stauffer BL, et al. Transgenic over-expression of YY1 induces pathologic cardiac hypertrophy in a sex-specific manner. Biochem Biophys Res Commun. 2015;462(2):131-137.

51. Lu Y, et al. Yin Yang 1 promotes hepatic steatosis through repression of farnesoid $\mathrm{X}$ receptor in obese mice. Gut. 2014;63(1):170-178.

52. Spiekerkoetter E, et al. Low-dose FK506 (Tacrolimus) in end-stage pulmonary arterial hypertension. Am J Respir Crit Care Med. 2015;192(2):254-257.

53. Tampe B, et al. Induction of Tet3-dependent epigenetic remodeling by low-dose hydralazine attenuates progression of chronic kidney disease. EBioMedicine. 2015;2(1):19-36.

54 . Haverty TP, et al. Characterization of a renal tubular epithelial cell line which secretes the autologous target antigen of autoimmune experimental interstitial nephritis. J Cell Biol. 1988;107(4):1359-1368. 